\title{
Small facial image dataset augmentation using conditional GANs based on incomplete edge feature input
}

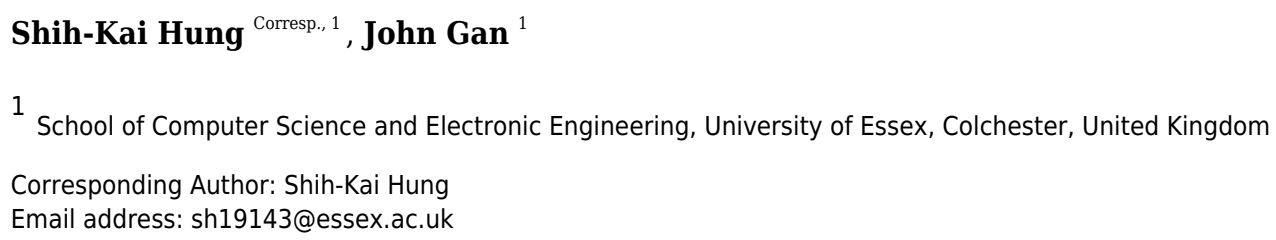

Image data collection and labelling is costly or difficult in many real applications. Generating diverse and controllable images using conditional generative adversarial networks (GANs) for data augmentation from a small dataset is promising but challenging as deep convolutional neural networks need a large training dataset to achieve reasonable performance in general. However, unlabeled and incomplete features (e.g., unintegral edges, simplified lines, hand-drawn sketches, discontinuous geometry shapes, etc.) can be conveniently obtained through pre-processing the training images and can be used for image data augmentation. This paper proposes a conditional GAN framework for facial image augmentation using a very small training dataset and incomplete or modified edge features as conditional input for diversity. The proposed method defines a new domain or space for refining interim images to prevent overfitting caused by using a very small training dataset and enhance the tolerance of distortions caused by incomplete edge features, which effectively improves the quality of facial image augmentation with diversity. Experimental results have shown that the proposed method can generate highquality images of good diversity when the GANs are trained using very sparse edges and a small number of training samples. Compared to the state-of-the-art edge-to-image translation methods that directly convert sparse edges to images, when using a small training dataset, the proposed conditional GAN framework can generate facial images with desirable diversity and acceptable distortions for dataset augmentation and significantly outperform the existing methods in terms of the quality of synthesised images, evaluated by Fréchet Inception Distance (FID) and Kernel Inception Distance (KID) scores. 


\section{Small facial image dataset augmentation using}

3 conditional GANs based on incomplete edge feature

4 input

\section{Shih-Kai Hung ${ }^{1}$, John Q. Gan ${ }^{1}$}

${ }^{1}$ School of Computer Science and Electronic Engineering, University of Essex, Colchester, UK

Corresponding Author:

Shih-Kai Hung ${ }^{1}$

Wivenhoe Park, Colchester, CO4 3SQ, UK

Email address: sh19143@essex.ac.uk

\section{Abstract}

Image data collection and labelling is costly or difficult in many real applications. Generating diverse and controllable images using conditional generative adversarial networks (GANs) for data augmentation from a small dataset is promising but challenging as deep convolutional neural networks need a large training dataset to achieve reasonable performance in general. However, unlabeled and incomplete features (e.g., unintegral edges, simplified lines, hand-drawn sketches, discontinuous geometry shapes, etc.) can be conveniently obtained through preprocessing the training images and can be used for image data augmentation. This paper proposes a conditional GAN framework for facial image augmentation using a very small training dataset and incomplete or modified edge features as conditional input for diversity. The proposed method defines a new domain or space for refining interim images to prevent overfitting caused by using a very small training dataset and enhance the tolerance of distortions caused by incomplete edge features, which effectively improves the quality of facial image augmentation with diversity. Experimental results have shown that the proposed method can generate high-quality images of good diversity when the GANs are trained using very sparse edges and a small number of training samples. Compared to the state-of-the-art edge-to-image translation methods that directly convert sparse edges to images, when using a small training dataset, the proposed conditional GAN framework can generate facial images with desirable diversity and acceptable distortions for dataset augmentation and significantly outperform the existing methods in terms of the quality of synthesised images, evaluated by Fréchet Inception Distance (FID) and Kernel Inception Distance (KID) scores. 


\section{Introduction}

41 Deep convolutional neural networks generally require a large amount of training data to improve accuracy on unseen data or testing data (Tan et al., 2018). For many practical applications, it is expensive to collect a large amount of training data for deep learning. Data augmentation is often used to generate more training data. Traditional approaches for data augmentation include geometric transformations, such as translation, scaling, flip, rotation, etc., especially for image data (Mikolajczyk \& Grochowski, 2018). However, the diversity introduced by traditional augmentation methods is limited and insufficient for many applications. The motivation of this work is to augment a small image dataset by making use of conditional edge features extracted from the available training images, and it can be expected that the synthesised images are more diverse and less distortive than those obtained from traditional methods. photorealistic images from conditional data, have become one of the most popular research fields in image synthesis. Conditional inputs, such as edges, mark points, masks, semantic maps, labels and so on, can be used to manipulate the images generated by GANs, making the synthesised images not only diverse but also controllable (Isola et al., 2017; Torrado et al., 2020). Image-toimage translation methods using conditional GANs directly learn the pixel mapping relationship between conditional edge features and real images (Lin et al., 2018; Wang \& Gupta, 2016). Although image-to-image methods based on conditional GANs have been developed for controllable image synthesis, there are still several problems that should be resolved when applying them on a small training dataset: 1) Compared with unconditional GANs, a limitation of conditional GANs is that the output images must be generated from the corresponding conditional inputs, hence a clear mapping relationship between input and output should be correctly established. Corresponding mapping relationships are hard to be discovered, especially when only a very small training dataset is available for deep neural networks to learn. 2) With a small training dataset, the training process for image-to-image translation is easy to converge but difficult to obtain high-quality results due to the overfitting problem and insufficient information about the underlying data distribution, whether the used conditional features are of high quality or not (Dimitrakopoulos, Sfikas \& Nikou, 2020), the discriminator will overfit the training data, and the generator would produce unexpected distortions in the generated images in the validation or application phase (Arjovsky \& Bottou, 2017; Gu et al., 2019). 3) Training GANs using a small dataset must deal with the inevitable problem of mode collapse (Zhao, Cong \& Carin, 2020), which implies that the GANs may learn the training data distribution from a limited number samples only but overlook other useful training data (Srivastava et al., 2017). Other issues, such as non-convergence and instability, would also worsen the quality of the generated images (Salimans et al., 2016). Clearly, it is a challenging task to synthesise photorealistic images using conditional GANs based on incomplete conditional features and a small number of training images.

Edge-based image-to-image translation using conditional GANs has the advantage of introducing diversity in image data augmentation, but it is challenging in terms of generating high-quality 
80 photorealistic images. Extracted edges cannot be regarded as perfect conditional features that 81 support various advanced visual tasks and contain all visual information of potential perceptual 82 relevance (Elder, 1999). Since edges generally contain incomplete information, such as

83 unintegral geometry, simplified lines, discontinuous shapes, missing components, and undefined

84 contours, it is hard for image-to-image translation methods to map edges to realistic images 85 without clear conditional information.

86 To simply demonstrate the impact of the number of training images and incomplete conditional 87 edges on the quality of the images generated using conditional GANs, some preliminary 88 experimental results are shown in Figure 1. It can be observed that the level of detail in the input 89 edge features and the number of training images have considerable influence on the quality of 90 both training and inference results, which is more serious on the inference results though because 91 the input edge features used in the inference process have not been used in the training process.

92

93

94

95

96

97

98

99

100

101

102

103

104

105

106

107

108

109

110

111

112

113

114

115

116

117

118

119
In this paper, a new image-to-image translation framework using conditional GANs is proposed, which can generate diverse photorealistic images from limited edge features after training with a small number of training images. Instead of deepening the convolutional layers or increasing the number of parameters, the proposed conditional GAN framework can learn additional relationships between incomplete edges and corresponding images, because regional binarisation and segmentation masks are used as new reference information, which can be obtained automatically by image processing. In particular, the proposed method can beneficially obtain extra pixel correlations between conditional edge inputs and the corresponding ground truth images to mitigate the influence of the overfitting problem during training. If the conditional GANs can efficiently learn from informative conditional inputs, such as colour, texture, edges, labels, etc., then it would be effortless to generate corresponding photorealistic image outputs (Dosovitskiy \& Brox, 2016; Wei et al., 2018). A new network structure is proposed in this paper to divide the image synthesis task into two stages: the first stage transforms conditional input with incomplete edges into refined images as the new conditional input for the second stage, whose pixel values are obtained by combining the information from segmentation masks and binarised images. The second stage transforms the refined images into photorealistic image outputs.

The experimental results have demonstrated that the proposed method can generate high-quality diverse images even with a very small training dataset and very sparse edge features as conditional input. In addition, the generated images do not contain large distortions from incomplete or modified edge inputs for data augmentation purposes. The contributions of this paper are as follows:

- In order to deal with the problem of distortions in the images generated by conditional GANs due to using very small training data and sparse conditional input for diverse data augmentation, a new conditional GAN framework has been proposed, which converts the original incomplete edges into new conditional inputs in an interim domain for refining images and thus alleviates distortions caused by small training data and incomplete conditional edges.

Peer] Comput. Sci. reviewing PDF | (CS-2021:08:64945:1:0:NEW 20 Sep 2021) 
120

121

122

123

124

125

126

127

128

129

130

131

132

133

134

135

136

137

138

139

140

141

142

143

144

145

146

147

148

149

150

151

152

153

154

155

156

157

158

- For the first time, the proposed method uses the mixture of pixel values of both binary images and segmentation masks to enhance the conditional input in an interim domain for refining images, which can integrate facial components, including eyes, nose, mouth, etc., so as to introduce diversity and enhance the quality of the images generated by conditional GANs trained using a very small training dataset.

- A facial image augmentation method using conditional GANs has been proposed, which can generate photorealistic facial images of diversity from incomplete edges or handdrawn sketches. Compared with existing edge-to-image translation methods without ideal conditional inputs, the proposed method is tolerant to various incomplete edges as conditional inputs and able to generate diverse images of higher quality in terms of Fréchet Inception Distance (FID) (Heusel et al., 2017) and Kernel Inception Distance (KID) (Binkowski et al., 2018).

This paper is organised as follows: In section 2, the related work is reviewed, including methods for image data augmentation, image-to-image translation, challenges in training GANs using small datasets. In section 3, the proposed methods, including the proposed conditional GAN framework, image pre-processing for generating the interim domain, and training strategies, are described in detail. Section 4 describes the experiment design, including data preparation and implementation details. The experimental results are presented and the performance of the proposed conditional GAN framework is evaluated qualitatively and quantitatively in section 5 . Finally, conclusions, limitations and future work suggestions are presented in section 6 .

\section{Related Work}

The method proposed in this paper aims to use incomplete or modified edge features to augment small facial image datasets. The method was based on image-to-image translation using conditional GANs. This section introduces related techniques for image synthesis using conditional GANs.

\section{Image Data Augmentation}

Image data augmentation is applied in many applications to increase dataset size and data diversity. Deep neural networks are not easy to be trained well using small training datasets due to overfitting problems (Bartlett et al., 2020). One of the solutions to overcome overfitting is training data augmentation, which intends to boost the diversity of a small training dataset (Shorten \& Khoshgoftaar, 2019). Traditional methods such as rotation, reflecting, translation, scaling, cropping, blurring, grey scaling and colour converting, have been commonly used for image data augmentation to reduce overfitting when training deep neural networks for image classification applications. Although these traditional techniques can produce similar images of high-quality, they rarely enlarge the feature diversity in the original images. Therefore, developing novel methods image data augumentation, which can synthesise not only diverse but also photorealistic images based on a small image dataset, is a very important but challenging task. 
159

160

161

162

163

164

165

166

167

168

169

170

171

172

173

174

175

176

177

178

179

180

181

182

183

184

185

186

187

188

189

190

191

192

193

194

195

196

197

198

199

Many GANs have been proposed for image synthesis (Gatys, Ecker \& Bethge, 2016; Iizuka, Simo-Serra \& Ishikawa, 2017). Since conditional GANs provide alternative methods to edit images as well as manipulate generative attributes, they have been applied to generate highquality images of diverse features (Lin et al., 2018a). Image synthesis using conditional GANs can boost the applications of data augmentation in many real applications, but it is hard to generate high-quality diverse images when the training dataset is small.

\section{Image-to-image Translation and Image Synthesis Using Conditional GANs}

Image-to-image translation is a type of image synthesis using conditional GANs with specific forms of conditions, such as videos and images (Azadi et al., 2018; Szeto et al., 2021), scenes (Ashual \& Wolf, 2019; Johnson, Gupta \& Li, 2018), or segmentation masks (Cherian \& Sullivan, 2018; Park et al., 2019). The conditional inputs can be transferred from a source domain to a target domain by using supervised learning techniques. The main concept of image-to-image translation is to learn the data mapping relationships (Liu, Breuel \& Kautz, 2017). Image-toimage translation methods can automatically generate images between the corresponding domains (Mo, Cho \& Shin, 2019) and discover the dependence with pairs of images to translate features into realistic images. Image-to-image translation methods provide a prominent approach to image synthesis with diverse results by using controllable conditional features (Isola et al., 2017; Zhu et al., 2017).

With remarkable advantages in image-to-image translation, edge-to-image synthesis has achieved visually pleasing performance (Yi et al., 2017). Compared with other conditional forms, edges are one of the most easy-to-obtain and simple features in computer vision, and hand-drawn sketches can be regarded as a specific form of edge features (Chen \& Hays, 2018). Since edges usually contain critical information, such as gradients, shapes, contours, profiles, boundaries and so on, they directly provide simple and direct depictions for objects (Chen et al., 2009; Eitz et al, 2011). The benefit of using edge features is that they are flexible to modify for introducing diversity in data augmentation. However, in contrast to labelled images, edges as conditional inputs often preserve insufficient information (e.g., textures, colours, brightness, labels, etc.), which makes it hard to generate desirable high-quality images using edge-to-image translation. Face synthesis requires integral contours that faithfully reflect the inputs and connections to the realistic context ( $L i$ et al., 2020). Incomplete features with missing parts or undefined components will affect the quality of images generated by conditional GANs ( $J o \&$ Park, 2019; Karras et al., 2018). To alleviate the influence of lacking ideal conditional inputs, this work proposes to reconstruct the missed features in the conditional input and transform limited edge information into refined images by introducing an interim domain to alleviate the negative effect of imperfect conditional features on the quality of the generated images.

\section{Challenges in Training GANs Using Small Training Dataset}

Mode collapse is a major problem in training GANs using small training data, which makes it fail to switch training samples and thus the trained GANs would be over-optimised with a limited number of training samples only (Odena, Olah \& Shlens, 2017). Due to the mode collapse problem, the generator is constructed with very limited training data, which makes the discriminator believe that the generative outputs are real instead of fake. This is one of the key 
200

201

202

203

204

205

206

207

208

209

210

211

212

213

214

215

216

217

218

219

220

221

222

223

224

225

226

227

228

229

230

231

232

233

234

235

236

237

238

causes that make it difficult for GANs to generate diverse results for data augmentation purposes (De Cao \& Kipf, 2018).

Another drawback in training GANs using a small training dataset is that it is hard to fine-tune all parameters to discover an optimal balance between the generator and discriminator. Both the generator and discriminator contain a large number of trainable parameters, which necessarily need enormous training data to prevent from overfitting problems (Gulrajani et al., 2017; Mescheder, Geiger \& Nowozin, 2018). Ideally, training a GAN requires a large amount of training data for optimising its parameters and reducing losses to generate photorealistic image outputs (Wang et al., 2018c). Therefore, reducing the requirement for a large amount of training data is a grand challenge in using GANs for image data augmentation in which available training data is limited. Our previous preliminary work (Hung \& Gan, 2021) partly addressed the above challenges by proposing a new conditional GAN architecture. This paper substantially extends our preliminary work via further investigation and deeper analysis of much more experimental results.

\section{Methods}

Image-to-image translation methods find specific mapping relationships between source distribution and target distribution. In general, a small number of paired features may not comprehensively align with the source and target distributions using imperfect conditional inputs such as incomplete edges and a small training image dataset. Therefore, data refining in paired features can be adopted to expand mapping relationships based on a small training dataset. In this section, a new method is proposed for facial image synthesis based on a very small training image dataset.

With incomplete conditional features in the source domain and small training data in the target domain, the mapping between source and target domains cannot be described by clear one-to-one relationships. The method proposed in this paper transfers the source domain to an interim domain for refining images with extra annotated information, in which newly defined images in the interim domain need to be generated based on a small training dataset. The interim domain can provide extra reference information to discover more mapping relationships between the source and target domains.

Figure 2 shows the proposed translation method using a small training dataset. It is difficult using a small training dataset to obtain a comprehensive view of correct mapping relationships between the source domain and target domain without sufficient representative training samples, as shown by the blue line in Figure 2. Even if changing the types of the conditional inputs, a similar situation remains as it is still difficult to learn correct mapping relationships, as demonstrated by the red line in Figure 2. For the purpose of comprehensively finding correct mapping relationships, extending the mapping relationships in an interim domain for refining images, as shown by the green dotted line in Figure 2, can reduce uncertainty caused by using a small training dataset and incomplete edge features as conditional inputs for data augmentation 
239 with diversity. This will be explained in more detail when introducing the proposed conditional 240 GAN framework later.

241 When training GANs using small training datasets, the following factors should be considered:

242 1) It is difficult to avoid distortions in the generated images and training imbalance with a small 243 number of training images or insufficient diverse samples. 2) Through deep convolutional neural 244 network structures, such as convolution, normalisation and downsampling, it is easy to lose 245 spatial information and impractical to completely preserve the conditional information with a 246 small number of training images (Kinoshita \& Kiya, 2020; Wang et al., 2018a). If the conditional 247 inputs contain sparse, unclear, limited, discontinuous, or incomplete features, fine-tuning model 248 parameters without distortions becomes much more difficult. 3) Using a small training dataset 249 and limited conditional features will make the training easy to overfit but hard to obtain realistic 250 results. Since many parameters in a deep convolutional neural network need to be fine-tuned, it 251 is impossible to optimise all the parameter values using a small training dataset in terms of the 252 generalisation ability of the trained deep neural network. To tackle the above problems in 253 254 training GANs using a small number of training images, several training strategies are adopted in the proposed method, which are described as follows:

Enlarging the diversity of source domain: The training of a GAN using a small training dataset can easily converge but frequently attain unrealistic inference results, mainly because of the overfitting problem. It is impossible for GANs to have a whole view of the target domain through training with a limited number of training images. For the goal of achieving photorealistic results, both the discriminator and generator should stay in an equilibrium balance. Increasing the data diversity and widening the mapping relationship between the source domain and target domain could help achieve the required balance between the generator and discriminator when using a small number of training images. In the proposed method, new reference information is created by image pre-processing, and the adoption of the interim domain for refining images can enlarge the diversity of the source domain.

266

Double translation: Double translation strategy aims to decrease the chance of mode collapse in single translation approach and reduce the impact of the uncertainty due to using incomplete edges as conditional input, so as to alleviate the distortions caused by training with a small number of training images. For generating additional reference information, the proposed method combines binary images and segmentation masks to generate refined images as conditional input in the next translation. In the first translation, refined images with annotated facial components are generated from incomplete edge features. This translation is conducted between the source domain and the interim domain. The second translation is conducted between the interim domain and target domain, which can successively learn from the possible distortions in the first stage to avoid or alleviate negative distortions in the final outputs.

275 lost during training in the convolutional neural layers, and the relationships between the source domain and target domain will become incomprehensive. In order to reduce the spatial information vanishing, edge features in the source domain can be reused in each translation. 
279 Freezing weights: Weight freezing is a strategy to overcome the gradient vanishing problem

280 during training, which often happens when using a small training dataset. If the provided training 281 data cannot give the discriminator enough information to progress the generator, the gradient will 282 become smaller and smaller when going from bottom to top layers of the network. Incomplete 283 conditions would worsen the gradient vanishing problem and make it impossible to fine-tune the 284 model parameters to obtain realistic results. Hence, freezing part of weights in separate training 285 stages allows the discriminator to acquire information from each training stage rather than tuning 286 all parameters at one time.

287 The Proposed Conditional GAN Framework

288 To mitigate the output distortions caused by using a small training dataset and incomplete edge 289 as conditional input, additional paired segmentation masks and regional binary images are used 290 as reference information in the proposed method, which can enrich the mapping relationships

291

292

293

294

295

296

297

298

299

300

301

302

303

304

305

306

307

308

309

310

311

312

313

314

315

316

317

318 between the source domain and target domain. Consequently, the proposed method creates additional data distributions from the small training dataset using image pre-processing, and the data in the interim domain provides more referable features than the original incomplete edges in the source domain.

Two U-nets (Ibtehaz \& Rahman, 2020; Ronneberger, Fischer \& Brox, 2015) are adopted in the proposed conditional GAN framework for image-to-image translation. This structure can achieve better performance when training conditional GANs using small training data for two reasons: on one hand, during the training process the U-nets create images based on the special concatenating structure, which is beneficial to retain the matched features from limited conditional features for integral perceptions in convolutional layers. On the other hand, the U-net structure is simple and beneficial to generate images without using very deep convolutional layers, which is critical to alleviate the gradient vanishing problem during training using small training data and incomplete edges. The proposed framework also reuses the conditional input information to strengthen the input features at each training stage, and freezing weights for separate networks at each training stage can prevent from gradient vanishing as well. To sum up, the proposed conditional GAN framework can alleviate the problems in training GANs using small training data by intensifying the conditional information in the source domain. An overview of the proposed conditional GAN framework is shown in Figure 3 and described as follows.

The proposed model consists of three parts: 1) image pre-processing, 2) generators and 3) discriminators. The two generators use the same convolutional structure of the U-net, both of which down-sample and then up-sample to the original size of input images. All convolutional layers use convolution kernels of size $3 \times 3$ (Yu et al., 2019), and normalisation is applied to all convolutional layers except for the input and output layers (Zhou \& Yang, 2019). In the training phase, the first generator is used to create refined images based on the original sparse edges and ground truth. The refined images are referred from image pre-processing, which contain features related to texture, colour, shape of different facial components. The second generator is designed to improve the synthetic process to generate photorealistic images from the interim domain. In 
319 the inference phase, the generators use the fine-tuned parameters to generate photorealistic

320

321

322

323

324

325

326

327

328

329

330

331

332

333

334

335

336

337

338

339

340

341

342

343

344

345

346

347

348

349

350

351

352

353

354

355

356

357

358

images from conditional edges that may have not been seen during training. The two discriminators have the same task of distinguishing between real and fake images: the first one is to identify generated images in terms of refined images, and the other is in terms of the ground truth.

\section{Image Pre-processing and Refining}

Image refining is essential for providing informative conditional features since incomplete edges may contain much unidentical information representing the same facial component. This uncertainty makes it difficult for conditional GANs to comprehensively find pixel relevance in different domains. For instance, an unclear "black circle" with incomplete edges can represent either nose, ear or eye, even if using a powerful network, it is difficult to learn well with a rare sign of "circle" as conditional input without any other crucial information (e.g., colour, types, angels, positions, textures, sub-components, brightness, layouts, shapes, etc.). A refining process can be employed within an image-to-image translation method, which enhances powerful one-toone mapping by providing close to ideal conditional input. However, there is no guarantee that ideal conditional inputs can be obtained in real applications, especially if the conditional inputs are incomplete or sparse edges. These uncertainties can result in unexpected distortions. If the interim domain for refining images can provide more specific information, the synthetic quality will be improved. Therefore, enhancing conditional information is one of the important goals of image pre-processing and refining.

Edge Extraction: Edges may contain incomplete features with many possible feature types, including undefined density, shape or geometry (Royer et al., 2017). However, to achieve high performance, image-to-image translation methods need clear conditions (Lee et al., 2019). In order to generate photorealistic images from limited conditional information, extending translation relationships based on proper reference images can make the mapping relationships between the source domain and target domain more precise based on a small training dataset. As an example, the corresponding mapping relationships among the ground truth, conditional features, and refined image are shown in Figure 4. The ground truth image is responsible for providing not only realistic features but also reference images to composite refined images. The red boxes shown in Figure 4 indicate the eye mapping in different domains, and the new relationships are expected to effectively reduce the uncertain mapping in image-to-image translation.

Adoption of an Interim Domain: In contrast to directly transforming the source edges to target results, the proposed conditional GAN framework converts conditional edges to refined images in an interim domain first. The interim domain trends to reconstruct possible missing information from incomplete conditional features using a U-net. Mode collapse problem may happen in the interim domain when incomplete edges are transferred to a refined image. Nevertheless, the translation at this stage is useful for facial component identification because the incomplete edges in the source domain are further processed. The refined images provide clearer accessorial information than the original incomplete edges, even if they are converted into simplified

Peer] Comput. Sci. reviewing PDF | (CS-2021:08:64945:1:0:NEW 20 Sep 2021) 
359

360

361

362

363

364

365

366

367

368

369

370

371

372

373

374

375

376

377

378

379

380

381

382

383

384

385

386

387

388

389

390

391

392

393

394

395

396

397

398

samples when mode collapse happens. By trial and error, appropriate regional features as reference images can reduce distortions and mismatch of features based on very limited edges. Therefore, the refined images are constructed by combining binarised images and segmentation masks, as shown in Figure 3. In short, the main function of the interim domain is to refine the original data distribution and strengthen the incomplete conditional features from the source domain.

Figure 5 (a) shows inference results of using uncertain edges to generate segmentation masks from 50 paired training images. To handle the incomplete edges in the conditional input, facial components can be reconstructed by a U-net in the interim domain. It is evident that the proposed conditional GAN can learn from only 50 segmentation masks to generate more integral face components, such as nose, eyebrow, hair and mouth. Figure 5 (b) illustrates examples where incorrect eye shapes are obtained, as shown in the red boxes, which would aggravate distortions in the target domain. What is worse, this situation is hard to be solved because it is difficult to increase the number of diverse samples based on a small dataset as GANs generally require more diverse data to be trained well. To resolve this problem, binary images with clear regional information are obtained through image pre-processing, which can enhance the contours and thus reduce distortions in facial components, as shown in Figure 4. In contrast to imprecisely depicting facial components in segmentation masks, binary images obtained by appropriate thresholding can produce more correct contours than segmentation masks and thus alleviate the problem caused by very limited training data.

Figure 6 shows that binary images can handle uncertain edge density in the inference phase to enhance crucial edge information with regional distributions. Binarised regional features can be extracted by the corresponding edge distribution from a small training dataset, which can not only integrate crucial contours, as shown in Figure 6 (a), but also get rid of meaningless noise when various untrained edges may be unrecognisable in the inference phase, as shown in Figure 6 (b). It is noteworthy that the results presented in Figures 5 and 6 can be regarded as those from an ablation study, which shows that removing the component of combining binarised regional features in the proposed method will significantly deteriorated the performance of the proposed conditional GAN.

\section{Model Training and Loss Functions}

Conditional Adversarial Loss: During training the proposed conditional GAN framework, it is difficult to find a balance between the generator and discriminator, especially when only very limited training data is available. Using an appropriate loss function is critical to ensure good quality of the generated images. Firstly, to distinguish real images from fake ones, the following basic loss function is used for the two convolutional neural networks, which is known as conditional adversarial loss.

$\mathcal{L}_{a d v}(D, G)=\mathbb{E}_{I, S}[\log D(S \mid I)]+\mathbb{E}_{I, I},\left[\log \left(1-D\left(I, G\left(I^{\prime} \mid I\right)\right)\right)\right]$

where $\mathbb{E}$ represents expected value, $G$ the generator, $D$ the discriminator, $S$ the source image, $I$ the conditional edge feature input, and $I^{\prime}$ the generated image. In the first U-net, $S$ should contain a mixture of pixels of binary image, segmentation mask and ground truth so as to distinguish 
399

400

401

402

403

404

405

406

407

408

409

410

411

412

413

414

415

416

417

418

419

420

421

422

423

424

425

426

427

428

429

430

431

432

433

434

435

436

437

between real refined image and fake generated image. In the second U-net, $S$ needs to be set as the ground truth only.

Feature Matching Loss: As in the pix2pix GAN model (Isola et al., 2017), the $L_{l}$ pixel loss as feature matching loss in the synthesised images is adopted. Since there are paired images in the training phase, the $L_{l}$ distance between the generated image $\left(I^{\prime}\right)$ and source image $(S)$ can be defined as follows:

$$
\mathcal{L}_{L 1}(G)=\mathbb{E}_{S, I, I^{\prime}}\left[\left\|S-G\left(I^{\prime} \mid I\right)\right\|_{1}\right]
$$

Overall Loss: The main purpose of using the loss function is to help the generator to synthesise photorealistic images by minimising the loss value with a limited number of input images. The overall loss function is defined as

$$
\min _{G} \max _{D} \mathcal{L}_{a d v}(D, G)+\alpha \mathcal{L}_{L 1}(G)
$$

where $\alpha$ is a weight parameter. A larger value of $\alpha$ encourages the generator to synthesise images less blurring in terms of $L_{l}$ distance.

The second U-net uses the refined images and original sparse lines as inputs to generate photorealistic images with the same loss function but different training parameters and freezing weights. Another difference between these two networks is the source image $S$, which should be either the refined images or the ground truth images.

\section{Experiments with the Proposed Conditional GAN Framework} Data Preparation

A small set of randomly chosen images from CelebA-HD (Liu et al., 2015) formed the training image dataset in our experiments. CelebA-HD includes 30,000 high-resolution celebrity facial images. All the images were resized to $256 \times 256$ for our proposed model. CelebAMask-HQ (Lee et al., 2020) is a face image dataset consisting of 30,000 high-resolution face images of size $512 \times 512$ and 19 classes, including skin, nose, eyes, eyebrows, ears, mouth, lip, hair, hat, eyeglass, earring, necklace, neck, cloth and so on. All the images in CelebAMask-HQ were selected from the CelebA-HD dataset, and each image has segmentation masks of facial attributes corresponding to CelebA-HD.

Since different numbers of segmentation masks were used to compare the performance of different methods with different numbers of training samples, the CelebAMask-HQ was used as the standard segmentation masks of reference images. If a very small training dataset is used, it would be fine to manually generate the segmentation masks by image pre-processing. In our experiments, the segmentation masks from CelebAMask-HQ were used as the common reference images of the corresponding training images.

\section{Implementation Details}

The hyper-parameter values were determined through trial and error in our experiments. For training the proposed conditional GAN framework, the Adam optimiser was used to minimise the loss function with the initial learning rate set to 0.0002 and the momentum 0.5 . The weight parameter in the loss function $\alpha$ was set to 100 . All the experiments were conducted on a desktop 
438

439

440

441

442

443

444

445

446

447

448

449

450

451

452

453

454

455

456

457

458

459

460

461

462

463

464

465

466

467

468

469

470

471

472

473

474

475

476

computer with NVIDIA GeForce RTX 2080 GPU, Intel Core i7-6700 (3.4GHz) processor, and 16G RAM.

Incomplete edges or hand-drawn sketches as conditional inputs usually represent abstract concepts, which are beneficial for generating diverse data augmentation results but it is difficult for conditional GANs to generate photorealistic images with limited conditional inputs based on small training data. In our experiments, edges were extracted by Canny edge detector (Canny, 1986), which can obtain simple and continuous edges using a set of intensity gradients from realistic images. Two intensity gradient magnitudes are used in Canny edge detector as a threshold range to control the edge density, which is determined in our experiments by a threshold ratio. It is the ratio of the high threshold value to the maximum magnitude, and the low threshold value is $40 \%$ of the high threshold value. The edges produced by the Canny edge detector are more similar to hand-drawn sketches than those by other commonly used edge detectors, as shown in Figure 7. The appropriate threshold ratio for the Canny edge detector was chosen through trial and error in our experiment. The red box in Figure 7 shows the edges extracted using the chosen threshold ratio, which has clear information about facial components without unexpected noise and meets the requirement for good conditional inputs.

In the design of the interim domain, the pixel values of the refined image were set by the following mixture ratios: $25 \%$ from binary image, $25 \%$ from segmentation mask, and 50\% from original image. Figure 8 shows the inference results of the refined images and the corresponding generated image outputs. The red boxes represent blending areas in the masks, binary images and texture features in the refined images, which reflect the brightness changes in the generated image outputs. The overlapped regions are visually darker and gloomier compared to other regions. Therefore, these blending areas from different reference images conduct transitions in brightness and lightness to synthesise realistic results. With the interim domain, the proposed conditional GAN can efficiently deal with both overlapped and non-overlapped mappings between segmentation masks and binary regions, leading to more photorealistic image outputs. Image blending with different styles is beneficial to augment training image datasets with diversity. In the proposed conditional GAN framework, generated images were controlled by conditional edge inputs. Exchanging or modifying edge features is an easy way to generate different images that increase data diversity and expand original facial features. Figure 9 shows examples of the generated images with facial features swapped on a small training dataset by exchanging edge components in conditional inputs. It can be seen that the generated images can preserve facial features with clear conditional edges and reconstruct the critical components in incomplete or undefined areas.

\section{Results and Performance Evaluation}

For performance evaluation, the proposed conditional GAN framework was used to generate images from different training images and conditional edge input settings. To demonstrate the performance of the proposed method, the state-of-the-art edge-to-image translation methods 
477 were compared both qualitatively by visual inspection of the quality and diversity of the

478 generated images and quantitatively in terms of FID and KID scores.

479 Diversity in Facial Image Augmentation Using the Proposed Conditional GAN

480 It is clear that the threshold ratio chosen for the Canny edge detector affects the density level of

481 the extracted edges, which as conditional inputs would affect the quality of the images generated

482 by the conditional GAN. It is desirable that the proposed conditional GAN can generate diverse

483 images with the change of edge density levels in the conditional input but be robust in terms of

484 the quality of the generated images. Figure 10 shows the inference results with different density

485 levels in the conditional edges, which were not included in the training phase except for those in

486 the red box. It can be seen that the generated images are slightly different with different density

487 levels in the conditional edge inputs and the distortions are small even when the GANs were

488 trained using a small dataset of 50 training images. The generated images are more photorealistic

489 when the conditional input contains less noise or unidentical edges, which correspond to those

490 generated with the edge density level chosen in the training phase, as shown in the red box.

491 Fortunately, with the change of the density levels of the conditional edge inputs, the quality of

492 the generated images is prevented from considerable deterioration because the refined images

493 can integrally represent facial features at an acceptable level based on a small dataset.

494 Consequently, as the conditional inputs to the second U-net in the second stage, they play an

495 important role in reducing distortions in the generated facial image outputs.

496 Figure 11 shows examples of facial image augmentation results using 50 training images to train

497 the proposed conditional GAN framework. Diverse new facial images can be generated from

498 each training image with extracted edges modified for desirable facial features as conditional

499 inputs. The modifications to the extracted edges include adding or deleting parts of the edges or

500 changing facial expression or direction, as shown in Figure 11. It can be seen that the image data

501 augmentation results using the proposed conditional GAN are more diverse than traditional

502 augmentation methods and the generated images are of good quality due to the use of the interim

503 domain. For data augmentation purposes, using deliberately modified edges as conditional inputs

504 to the proposed conditional GAN framework can boost the data diversity on the basis of the

505 available small set of training images.

506 Figure 12 shows some other examples of facial image data augmentation using 50 training

507 images to train the proposed conditional GAN, with edge features from multiple training images

508 swapped as conditional inputs. The red boxes in the figure indicate the original training images,

509 and the other images in a row are generated by the proposed conditional GAN, which shows

510 swapped facial features (including eyes, eyebrows, nose and mouth) or hairstyles. It can be seen

511 that by using mixed edge features from multiple training images as conditional inputs the

512 proposed conditional GAN can efficiently generate diverse facial images of good quality.

513 In general, it is difficult for image-to-image translation methods to generate high-quality images

514 with conditional inputs that are not directly corresponding to features in the training images, such

515 as hand-drawn sketches. In the previous experiments, it has been demonstrated that the interim

516 domain is helpful to generate high-quality images with various edge density levels for diversity. 
517 In our experiments, hand-drawn sketches were also used as conditional inputs for the proposed

518 conditional GAN to generate facial images with specific facial expressions or features. Figure 13

519 shows the inference results with hand-drawn sketches as conditional inputs, with the proposed

520 conditional GAN trained using a dataset of 50 training images. It is obvious that, when giving

521 unidentical or incomplete facial contours in the conditional inputs, the refined images generated

522 by the first U-net in the proposed conditional GAN structure are responsible for reducing

523 distortions in the generated images while keeping the diverse facial expressions introduced by

524 the hand-drawn sketches.

525 Qualitative Comparison

526 To evaluate the quality of the images generated by the proposed conditional GAN framework,

527 the images generated by the proposed method were compared with those generated by the state-

528 of-the-art edge-to-image translation methods, including pix2pix (Isola et al, 2017) and

529 pix2pixHD (Wang et al., 2018a), under the same training conditions and in terms how the

530 generated images are comparable to the ground truth images. Figure 14 shows representative

531 images generated respectively by the three conditional GANs for comparison, trained using the

532 same small dataset of 50 training images. Different sparse edges as conditional inputs were

533 tested. The results in Figure 14 demonstrate that the method proposed in this paper can generate

534 more photorealistic facial images with fewer distortions than pix2pix and pix2pixHD, when the

535 GANs were trained using small training data.

536 Quantitative Comparison

537 Since the number of training images is small, the difference between the generated images and

538 the corresponding ground truth images is noticeable by visual inspection. In order to

539 quantitatively compare the quality of the images generated by different conditional GANs, FID

540 and KID scores were adopted to evaluate the photorealistic scales of the generated images in our

541 further experiments. FID is widely adopted to evaluate the visual quality of generated images,

542 which calculates the Wasserstein distance between the generated images and the corresponding

543 ground truth images. KID can be used similarly for image quality measurement, but KID scores

544 are based on an unbiased estimator with a cubic kernel (Binkowski et al., 2018). Clearly, lower

545 FID and KID scores represent a better match between the generated images and the

546 corresponding ground truth images.

547 To evaluate the effect of the interim domain adopted in the proposed conditional GAN

548 framework on the quality of the generated images, the performance of double U-nets is

549 compared with that of a single U-net in terms of FID and KID with different threshold ratios

550 used in the Canny edge detector. In the training phase, one input type with threshold ratio $=0.4$

551 and three input types with threshold ratios $=0.2,0.4$ and 0.6 were considered and 50 training

552 images were used, whilst in the inference phase 11 different threshold ratios $(0.01,0.05,0.1,0.2$,

$5530.3,0.4,0.5,0.6,0.7,0.8$ and 0.9$)$ were tested with 1000 generated images respectively. Figure

55415 shows the comparative results in terms of FID and KID scores. Three points can be made

555 from the experimental results presented in this figure: Firstly, double U-nets achieved lower FID

556 and KID scores than single U-net, indicating that the interim domain for refining images in the 
557 proposed conditional GAN can reduce distortions caused by incomplete conditional edges and

558

559

560

561

562

563

564

565

566

567

568

569

570

571

572

573

574

575

576

577

578

579

580

581

582

583

584

585

586

587

588

589

590

591

592

593

594

595

small training dataset, and thus improve the quality of the generated images; Secondly, training with three conditional edge density levels, compared with only one conditional type, can achieve better and robust performance with various levels of conditional edge density (for better diversity); Thirdly, the most photorealistic performance was achieved when the edge density levels in the inference phase are close to those used in the training phase. The results presented in Figure 15 can be interpreted from the perspective of ablation study because removing the interim domain adopted in the proposed method will considerably deteriorate the performance of the conditional GAN.

This paper aims to generate photorealistic facial images using conditional GANs trained with a small set of training images for data augmentation. To evaluate the effect of the number of training images on the quality of the images generated by the proposed conditional GAN, pix2pix and pix2pixHD, different numbers $(25,50,100$, and 500) of training images were used to train each of the three conditional GANs separately. Moreover, in order to demonstrate the effect of different conditional edge density levels, both sparse edges (threshold ratio $=0.4$ ) and dense edges (threshold ratio $=0.2$ ) were used to generate 1000 images by each trained conditional GAN. The FID and KID scores of the images generated by the three conditional GANs were calculated respectively. Figure 16 shows the changes of FID and KID scores with the different numbers of training images, from which the following three points can be made: 1) With the interim domain, the proposed conditional GAN achieved lower FID and KID scores than pix2pix and pix2pixHD when trained with the same number of training images. 2) Dense conditional edges achieved lower KID and FID scores than sparse edges, but the diversity in the generated images may be constrained. 3) With the increase in the number of training images, the advantage of the proposed method over the existing methods becomes less obvious. This tendency indicates that the proposed conditional GAN framework is very effective when it is trained with a small number training samples and its performance would approach to that of the existing methods when the number of training samples becomes relatively large.

\section{Conclusions and Future Work}

In this paper, a new conditional GAN framework is proposed for edge-to-image translation based on a small set of training data, which can synthesise photorealistic diverse facial images using incomplete edges as conditional inputs for data augmentation purposes. In order to solve the problem in training conditional GANs using small training data, an interim domain for refining images is introduced in the proposed conditional GAN, which can effectively reduce unexpected distortions and thus improve the quality of the generated images. Experimental results have demonstrated that blending segmentation masks and regional binary images as refined reference images can reduce distortions in facial components of the images generated by the conditional GAN trained with a small training dataset. Compared with the existing edge-to-image translation methods, the proposed conditional GAN can not only automatically transfer incomplete 
596 conditional edges to reference images with more facial features in the interim domain but also 597 effectively reduce unexpected distortions caused by small training data.

598 Compared to directly transferring source domain into target domain, the proposed method can 599 have a more comprehensive view to generate more photorealistic edge-to-image translation 600 results when using various incomplete conditional edges for data augmentation. More 601 informative reference images can be constructed in the interim domain from incomplete edge 602 inputs to integrate useful facial components. The proposed conditional GAN trained using a 603 small dataset can synthesise various photorealistic facial images by manipulating conditional 604 edge features or using hand-drawn facial sketches for diverse image data augmentation.

605 Compared to the existing conditional GANs for image-to-image translation, the images

606 generated by the proposed conditional GAN have less distortion and more diversity, which is

607 desirable for data augmentation purposes.

608 Due to limited GPU computing facilities available for conducting our experiments, it is hard to 609 optimise the hyperparameters of the tested models, and the performance evaluation is based on 610 the comparison with two state-of-the-art methods only. More extensive comparative study would 611 be desirable in the future research to draw more reliable conclusions.

612 The advantage of the proposed conditional GAN framework over the existing methods becomes

613 less obvious when the number of training samples are relatively large. For future work, the

614 interim domain could be improved so that the proposed conditional GAN framework would also 615 significantly outperform existing methods for image data augmentation when a reasonably large 616 number of training image dataset is available.

617

\section{References}

619 Arjovsky M, Bottou L. 2017. Towards principled methods for training generative adversarial 620

621

622

623

624

625

626

627

628

629

630

631

632

633

634

635 networks. In: Proceedings of the international conference on learning representations, 1 6.

Ashual O, Wolf L. 2019. Specifying object attributes and relations in interactive scene generation. In: Proceedings of the IEEE international conference on computer vision, 4561-4569.

Azadi S, Fisher M, Kim V, Wang Z, Shechtman E, Darrell T. 2018. Multi-content GAN for few-shot font style transfer. In: Proceedings of the IEEE conference on computer vision and pattern recognition, 7564-7573.

Bartlett PL, Long PM, Lugosi G, Tsigler A. 2020. Benign overfitting in linear regression. National Academy of Sciences 117(48):30063-30070 DOI 10.1073/pnas.1907378117.

Binkowski M, Sutherland D, Arbel M, Gretton A. 2018. Demystifying MMD GANs. In: Proceedings of the international conference on learning representations, 1-36.

Canny J. 1986. A computational approach to edge detection. IEEE Transactions on Pattern Analysis and Machine Intelligence 8(6):679-698 DOI 10.1109/TPAMI.1986.4767851.

Chen T, Cheng M-M, Tan P, Shamir A, Hu S-M. 2009. Sketch2photo: Internet image montage. ACM Transactions on Graphics 28(124):1-10 DOI 10.1145/1618452.1618470. 
636 Chen W, Hays J. 2018. SketchyGAN: Towards Diverse and Realistic Sketch to Image

637

638

639

640

641

642

643

644

645

646

647

648

649

650

651

652

653

654

655

656

657

658

659

660

661

662

663

664

665

666

667

668

669

670

671

672

673

674

675

Synthesis. In: Proceedings of the IEEE conference on computer vision and pattern recognition, 9416-9425 DOI 10.1109/CVPR.2018.00981.

Cherian A, Sullivan A. 2018. Sem-GAN: Semantically-consistent image-to-image translation. In: Proceedings of the IEEE winter conference on applications of computer vision, 17971806 DOI 10.1109/WACV.2019.00196.

De Cao N, Kipf T. 2018. MolGAN: An implicit generative model for small molecular graphs. In: ICML 2018 workshop on theoretical foundations and applications of deep generative models DOI 10.26434/chemrxiv.14569545.v3.

Dimitrakopoulos P, Sfikas G, Nikou C. 2020. ISING-GAN: Annotated data augmentation with a spatially constrained generative adversarial network. In: IEEE 17th international symposium on biomedical imaging, 1600-1603 DOI 10.1109/ISBI45749.2020.9098618.

Dosovitskiy A, Brox T. 2016. Generating images with perceptual similarity metrics based on deep networks. In: Proceedings of the neural information processing systems conference, 658-666.

Eitz M, Richter R, Hildebrand K, Boubekeur T, Alexa M. 2011. Photosketcher: Interactive sketch-based image synthesis. IEEE Computer Graphics and Applications 31(6):56-66 DOI 10.1109/MCG.2011.67.

Elder J. 1999. Are edges incomplete? International Journal of Computer Vision 34:97-122 DOI 10.1023/A:1008183703117.

Gatys LA, Ecker AS, Bethge M. 2016. Image style transfer using convolutional neural networks. In: Proceedings of the IEEE conference on computer vision and pattern recognition, 2414-2423 DOI 10.1109/CVPR.2016.265.

Gu S, Bao J, Yang H, Chen D, Wen F, Yuan L. 2019. Mask-guided portrait editing with conditional GANs. In: Proceedings of the IEEE conference on computer vision and pattern recognition, 3436-3445 DOI 10.1109/CVPR.2019.00355.

Gulrajani I, Ahmed F, Arjovsky M, Dumoulin V, Courville AC. 2017. Improved training of Wasserstein GANs. In: Proceedings of the neural information processing systems conference, 5767-5777.

Heusel M, Ramsauer H, Unterthiner T, Nessler B, Hochreiter S. 2017. GANs trained by a two time-scale update rule converge to a local Nash equilibrium. In: Proceedings of the neural information processing systems conference, 6626-6637.

Hung SK, Gan QJ. 2021. Facial image augmentation from sparse line features using small training data. In: Proceedings of the International Work-Conference on Artificial and Natural Neural Networks. 547-558. DOI 10.1007/978-3-030-85030-2_45

Ibtehaz N, Rahman MS. 2020. MultiResUNet: Rethinking the U-Net architecture for multimodal biomedical image segmentation. Neural Networks 121:74-87 DOI 10.1016/j.neunet.2019.08.025.

Iizuka S, Simo-Serra E, Ishikawa H. 2017. Globally and locally consistent image completion. ACM Transactions on Graphics 36(4):107-110 DOI 10.1145/3072959.3073659.

Peer] Comput. Sci. reviewing PDF | (CS-2021:08:64945:1:0:NEW 20 Sep 2021) 
676 Isola P, Zhu J-Y, Zhou T, Efros AA. 2017. Image-to-image translation with conditional

677

678

679

680

681

682

683

684

685

686

687

688

689

690

691

692

693

694

695

696

697

698

699

700

701

702

703

704

705

706

707

708

709

710

711

712

713

714 adversarial networks. In: Proceedings of the IEEE conference on computer vision and pattern recognition, 1125-1134 DOI 10.1109/CVPR.2017.632.

Jo Y, Park J. 2019. SC-FEGAN: Face editing generative adversarial network with user's sketch and color. In: Proceedings of the IEEE international conference on computer vision, 17451753 DOI 10.1109/ICCV.2019.00183.

Johnson J, Gupta A, Li F-F. 2018. Image generation from scene graphs. In: Proceedings of the IEEE conference on computer vision and pattern recognition, 1219-1228 DOI 10.1109/CVPR.2018.00133.

Karras T, Aila T, Laine S, Lehtinen J. 2018. Progressive growing of GANs for improved quality, stability, and variation. In: Proceedings of the international conference on learning representations.

Kinoshita Y, Kiya H. 2020. Fixed smooth convolutional layer for avoiding checkerboard artifacts in CNNs. In: Proceedings of IEEE international conference on acoustics, speech, and signal processing, 3712-3716.

Lee C-H, Liu Z, Wu L, Luo P. 2020. MaskGAN: Towards diverse and interactive facial image manipulation. In: Proceedings of the IEEE conference on computer vision and pattern recognition, 5549-5558.

Lee H-Y, Tseng H-Y, Mao Q, Huang J-B, Lu Y-D, Singh M, Yang M-H. 2019. DRIT++: Diverse image-to-image translation via disentangled representations. Computing Research Repository 1905:1-14 DOI 10.1007/s11263-019-01284-Z.

Li Y, Chen X, Yang B, Chen Z, Cheng Z, Zha Z-J. 2020. Deepfacepencil: Creating face images from freehand sketches. In: Proceedings of the ACM international conference on multimedia, 991- 999 DOI 10.1145/3394171.3413684.

Lin J, Xia Y, Qin T, Chen Z, Liu TY. 2018. Conditional image-to-image translation. In: Proceedings of the IEEE conference on computer vision and pattern recognition, 55245532 DOI 10.1109/CVPR.2018.00579.

Liu M-Y, Breuel T, Kautz J. 2017. Unsupervised image-to-image translation networks. In: Proceedings of the neural information processing systems conference, 700-708 DOI 10.1109/BigComp48618.2020.00-92.

Liu Z, Luo P, Wang X, Tang X. 2015. Deep learning face attributes in the wild. In: Proceedings of the IEEE international conference on computer vision, 3730-3738 DOI 10.1109/ICCV.2015.425.

Mescheder L, Geiger A, Nowozin S. 2018. Which training methods for GANs do actually converge. In: Proceedings of the international conference on machine learning, 34783487.

Mikolajczyk A, Grochowski M. 2018. Data augmentation for improving deep learning in image classification problem. In: Proceedings of the international interdisciplinary PhD workshop, 117-122 DOI 10.1109/IIPHDW.2018.8388338. 
715 Mo S, Cho M, Shin J. 2019. InstaGAN: Instance-aware image-to-image translation. In:

$716 \quad$ Proceedings of the international conference on learning representations.

717 Odena A, Olah C, Shlens J. 2017. Conditional image synthesis with auxiliary classifier GANs.

718 In: Proceedings of the international conference on machine learning, 2642-2651.

719 Park T, Liu M-Y, Wang T-C, Zhu J-Y. 2019. Semantic Image Synthesis with Spatially-

720

721

722

723

724

725

726

727

728

729

730

731

732

733

734 Adaptive Normalization. In: Proceedings of the IEEE conference on computer vision and pattern recognition, 2337-2346 DOI 10.1109/CVPR.2019.00244.

Ronneberger O, Fischer P, Brox T. 2015. U-net: Convolutional networks for biomedical image segmentation. In: Proceedings of the medical image computing and computer-assisted intervention, 234-241 DOI 10.1007/978-3-319-24574-4_28.

Royer A, Bousmalis K, Gouws S, Bertsch F, Moressi I, Cole F, Murph, K. 2017. XGAN: Unsupervised image-to-image translation for many-to-many mappings. Computing Research Repository 1711:1-19 DOI 10.1007/978-3-030-30671-7_3.

Salimans T, Goodfellow I, Zaremba W, Cheung V, Radford A, Chen X. 2016. Improved techniques for training GANs. In: Proceedings of the neural information processing systems conference, 2234-2242.

Shorten C, Khoshgoftaar TM. 2019. A survey on image data augmentation for deep learning. Journal of Big Data 6(1):60 DOI 10.1186/s40537-019-0197-0.

Srivastava A, Valkoz L, Russell C, Gutmann MU, Sutton C. 2017. VEEGAN: Reducing

735 mode collapse in GANs using implicit variational learning. In: Proceedings of the neural information processing systems conference, 3308-3318.

736

737

738

739

740

741

742

743

744

745

746

747

748

749

750

751

752

753

Szeto R, El-Khamy M, Lee J, Corso JJ. 2021. HyperCon: Image-to-video model transfer for video-to-video translation tasks. In: Proceedings of the IEEE winter conference on applications of computer vision, 3080-3089.

Tan C, Sun F, Kong T, Zhang W, Yang C, Liu C. 2018. A survey on deep transfer learning. In: Proceedings of the 27th international conference on artificial neural networks, 270 279.

Torrado RR, Khalifa A, Green MC, Justesen N, Risi S, Togelius J. 2020. Bootstrapping conditional GANs for video game level generation. In: Proceedings of the IEEE conference on games, 41-48 DOI 10.1109/CoG47356.2020.9231576.

Wang T-C, Liu M-Y, Zhu J-Y, Tao A, Kautz J, Catanzaro B. 2018a. High-resolution image synthesis and semantic manipulation with conditional GANs. In: Proceedings of the IEEE conference on computer vision and pattern recognition, 1-13 DOI 10.1109/CVPR.2018.00917.

Wang X, Gupta A. 2016. Generative image modeling using style and structure adversarial networks. In: Proceedings of the European conference on computer vision, 318-335 DOI 10.1007/978-3-319-46493-0_20.

Wang X, Yu K, Dong C, Loy CC. 2018b. Recovering realistic texture in image superresolution by deep spatial feature transform. In: Proceedings of the IEEE conference on

754 computer vision and pattern recognition, 606-615. 
755

756

757

758

759

760

761

762

763

764

765

766

767

768

769

770

771

772

773

774

775

776

777
Wang Y, Wu C, Herranz L, van de Weijer J, Gonzalez-Garcia A, Raducanu B. 2018c. Transferring GANs: Generating images from limited data. In: Proceedings of the European conference on computer vision, 218-234.

Wei X, Gong B, Liu Z, Lu W, Wang L. 2018. Improving the improved training of Wasserstein GANs: A consistency term and its dual effect. In: Proceedings of the international conference on learning representations.

Yi Z, Zhang H, Tan P, Gong M. 2017. DualGAN: Unsupervised dual learning for image-toimage translation. In: Proceedings of the IEEE conference on computer vision and pattern recognition, 2849-2857 DOI 10.1109/ICCV.2017.310.

Yu J, Lin Z, Yang J, Shen X, Lu X, Huang TS. 2019. Freeform image inpainting with gated convolution. In: Proceedings of the IEEE international conference on computer vision, 4471-4480 DOI 10.1109/ICCV.2019.00457.

Zhao M, Cong Y, Carin L. 2020. On leveraging pretrained GANs for generation with limited data. In: Proceedings of the international conference on machine learning 119:1134011351.

Zhou X-Y, Yang G-Z. 2019. Normalization in training U-Net for 2D biomedical semantic segmentation. IEEE Robotics and Automation Letters 4(2):1792-1799 DOI 10.1109/LRA.2019.2896518.

Zhu J, Shen Y, Zhao D, Zhou B. 2020. In-domain GAN inversion for real image editing. In: Proceedings of the European conference on computer vision, 592-608.

Zhu J-Y, Park T, Isola P, Efros AA. 2017. Unpaired image-to-image translation using cycleconsistent adversarial networks. In: Proceedings of the IEEE conference on computer vision and pattern recognition, 2223-2232 DOI 10.1109/ICCV.2017.244. 


\section{Figure 1}

Examples of training results (left side) and inference results (right side) with a different number of training images and different conditional edge inputs by using the same parameter setting for the image-to-image translation method.
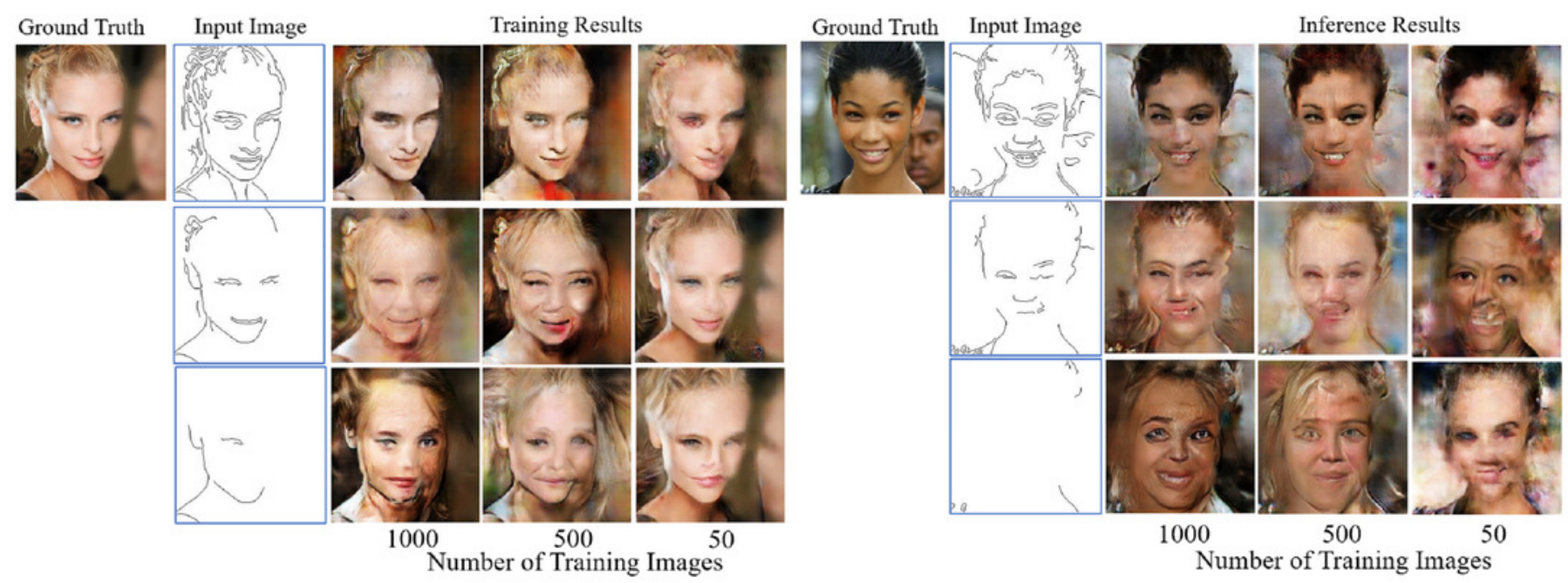


\section{Figure 2}

The proposed translation method by defining an interim domain for refining images based on a small training dataset.

Image pre-processing is adopted in the proposed conditional GAN to enhance the mapping relationship from source domain to target domain.

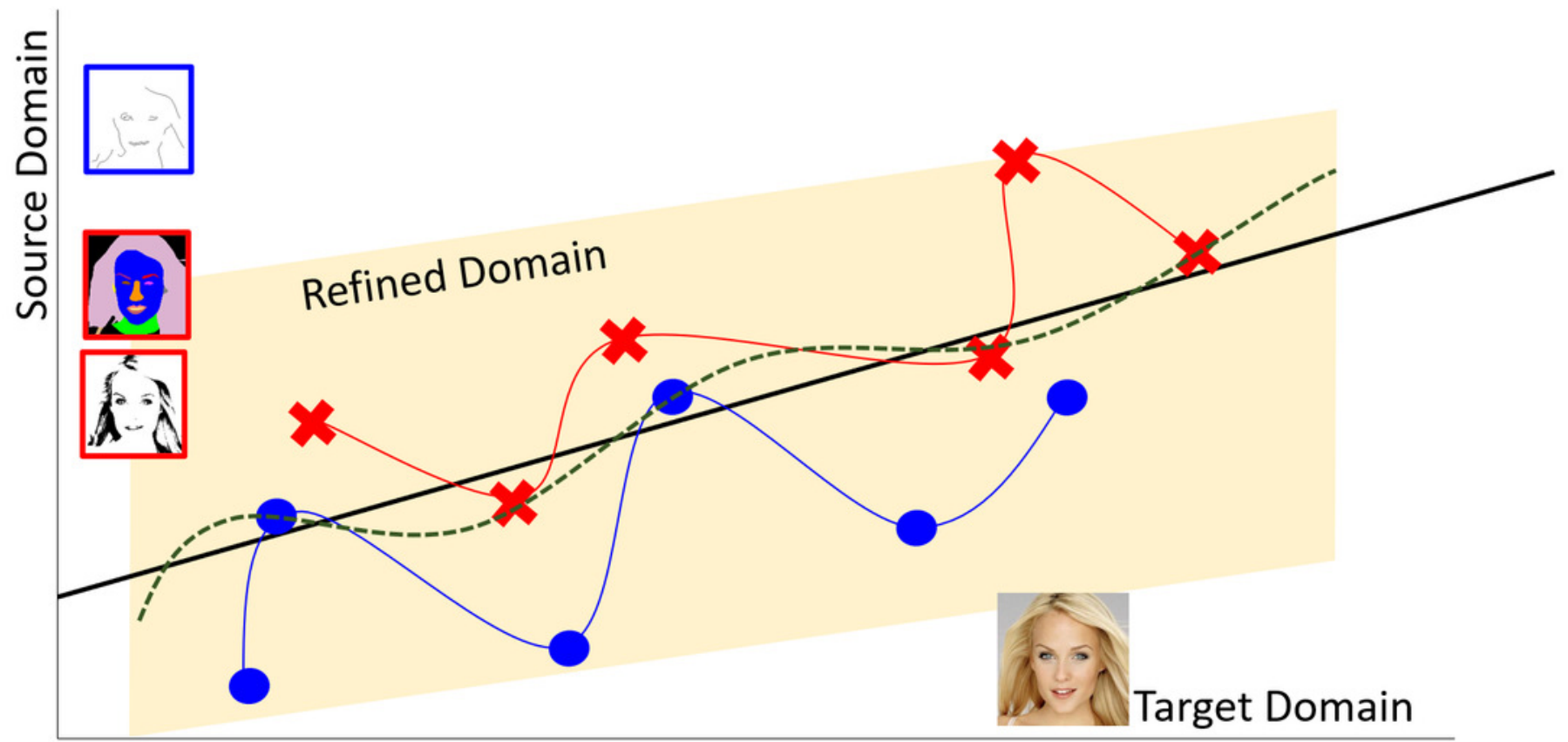

Ideal Mapping Relationship

- Edge image Mapping

*- Pre-processed Image Mapping _- - - Refined Image Mapping 
Figure 3

Overview of the proposed conditional GAN for translating edges to photorealistic images using two U-nets.

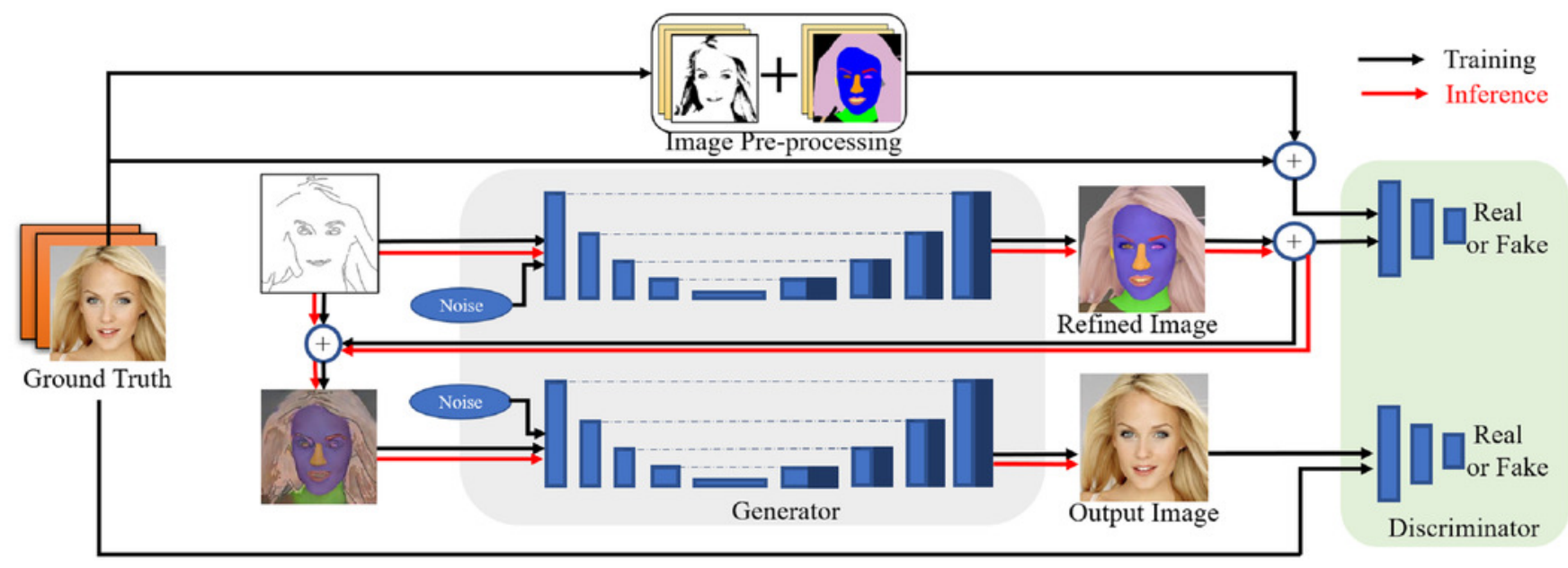


Figure 4

Corresponding mapping relationships among the conditional inputs, refined image and ground truth.

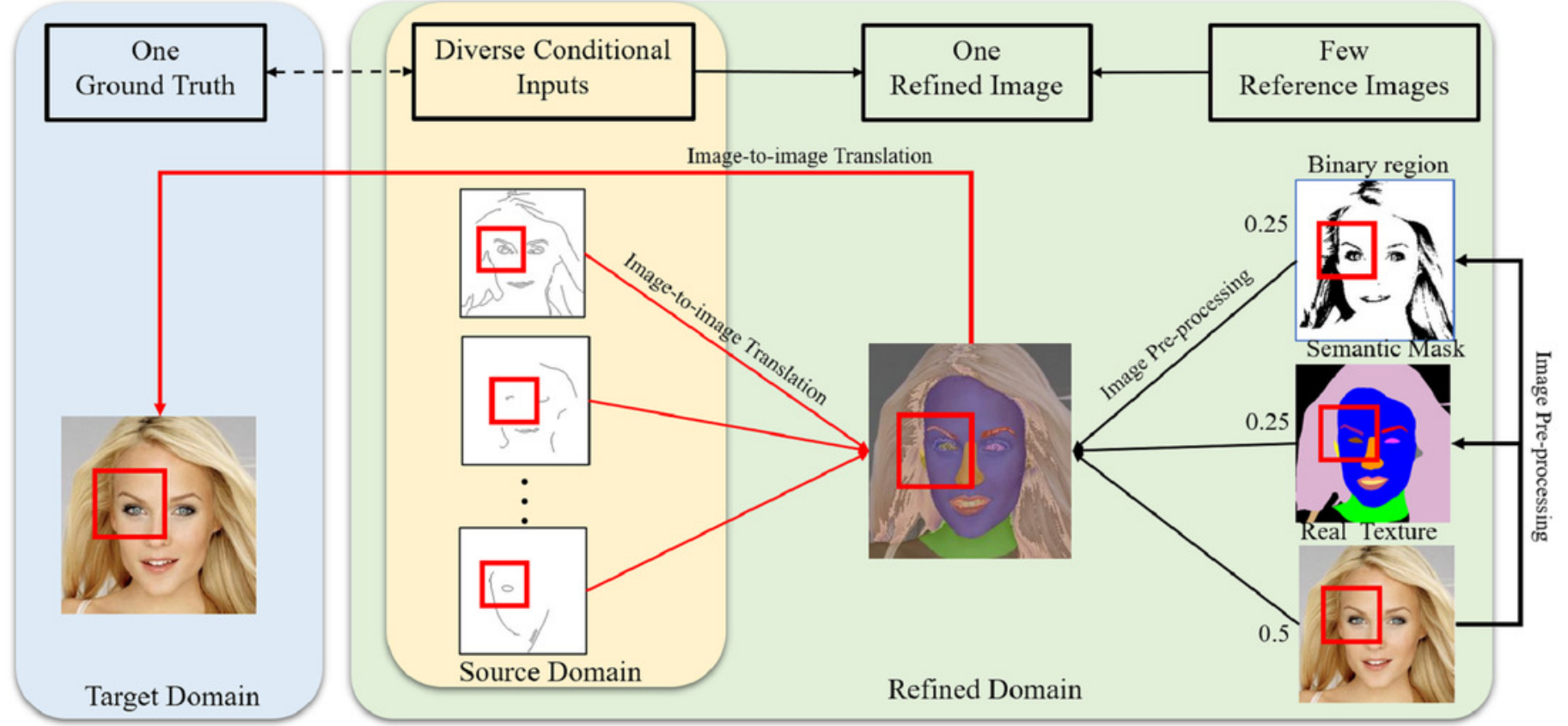




\section{Figure 5}

Inference results in translating sparse edges to labelled segmentation masks with 50 training images.

(a) The outputs can roughly resume the missing facial components from incomplete layouts when given abstract inputs. (b) The red boxes indicate the corresponding indefinite contours in the original inputs and generated masks.

(a)
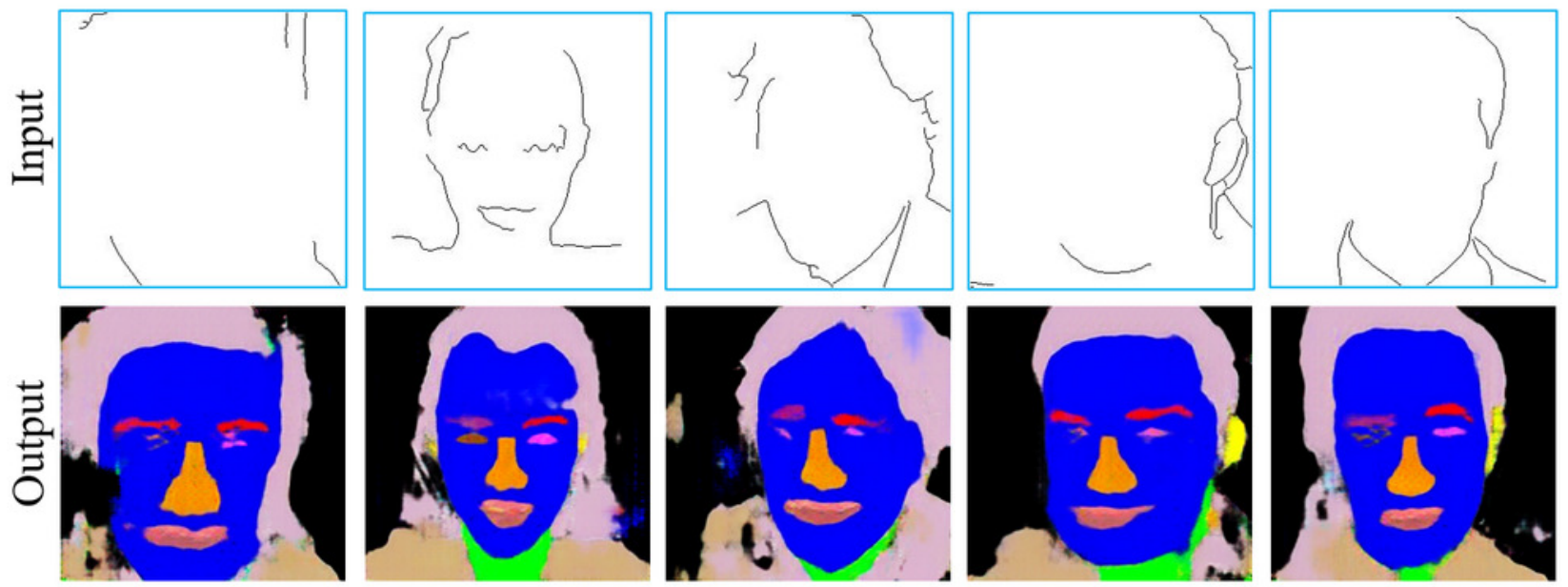

(b)
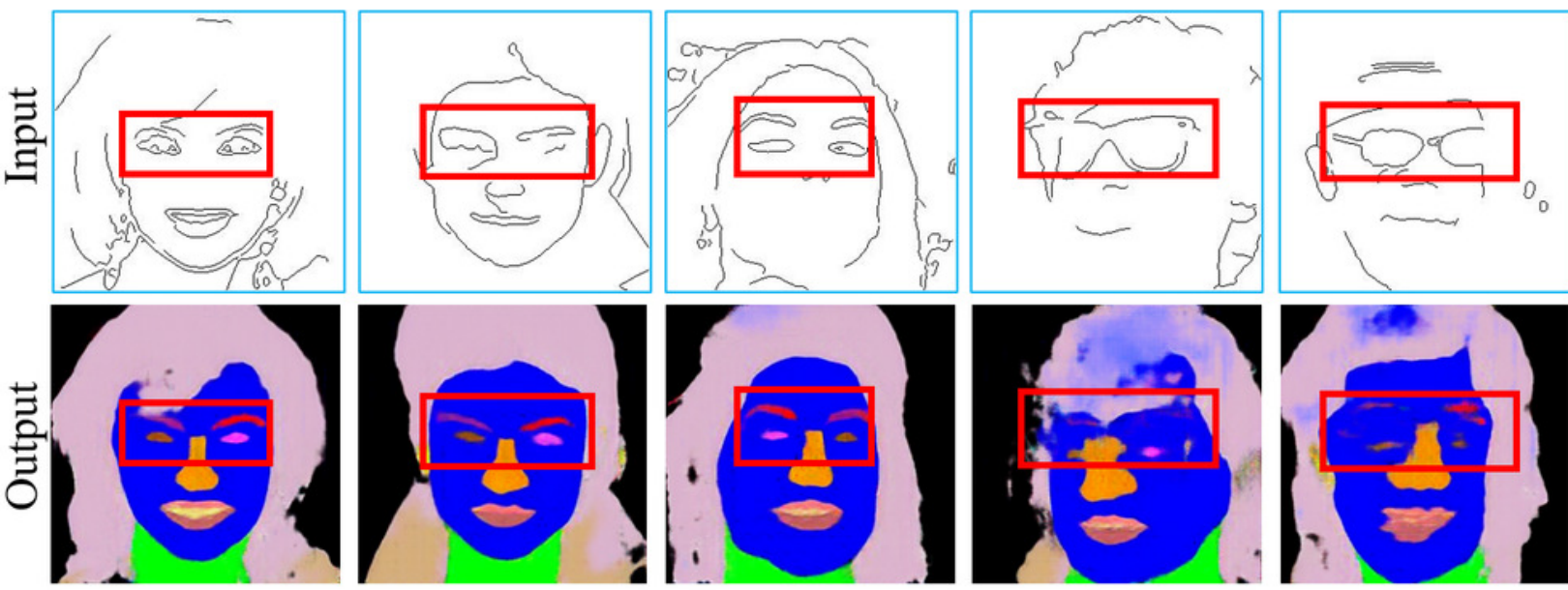
Figure 6

Inference results in translating sparse edges to binary regional images with 50 training images.

(a) The outputs can integrate discontinued contours when given sparse inputs. (b) The outputs can get rid of 'bogus' edges when given very dense inputs.

(a)
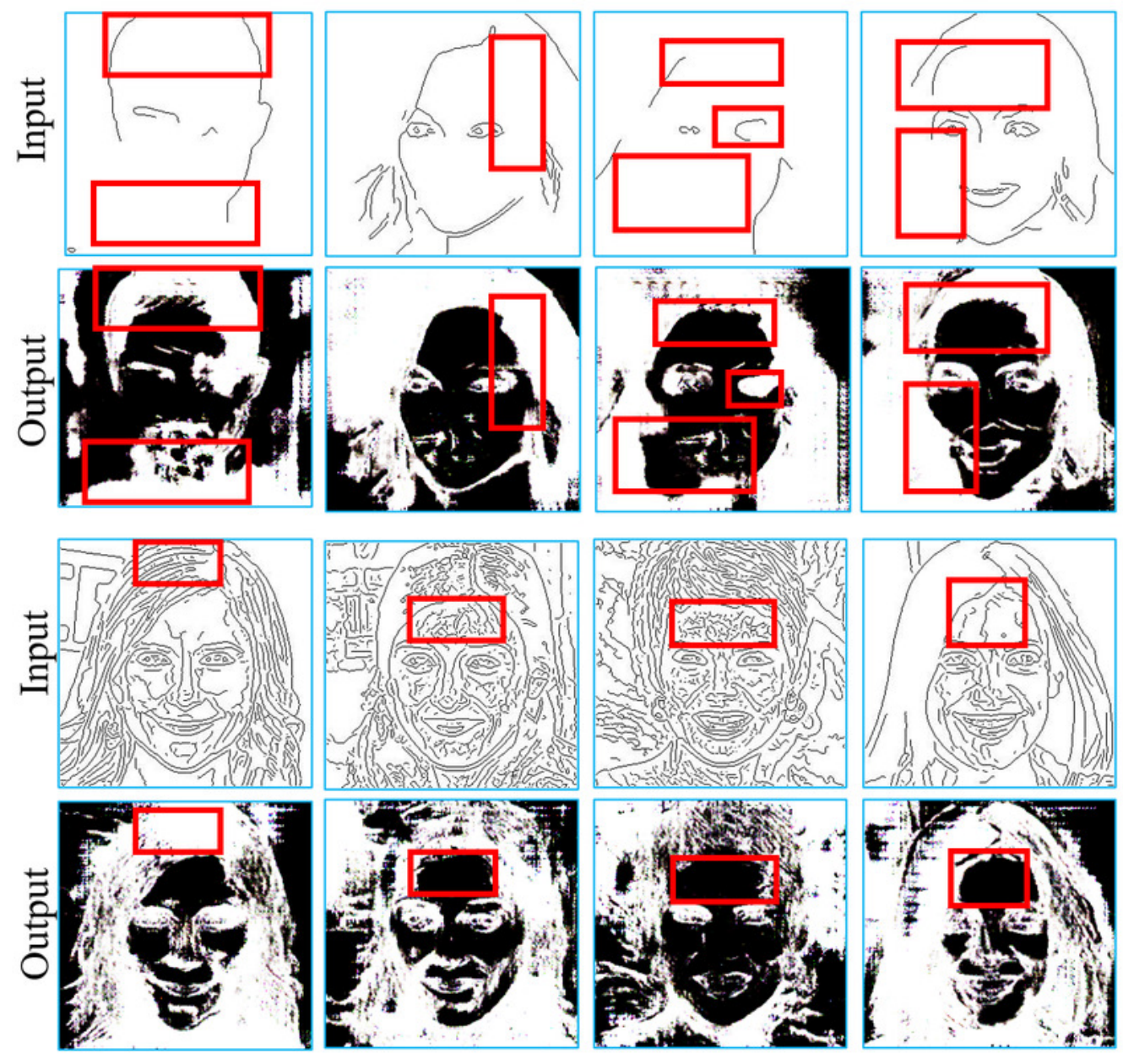

(b)
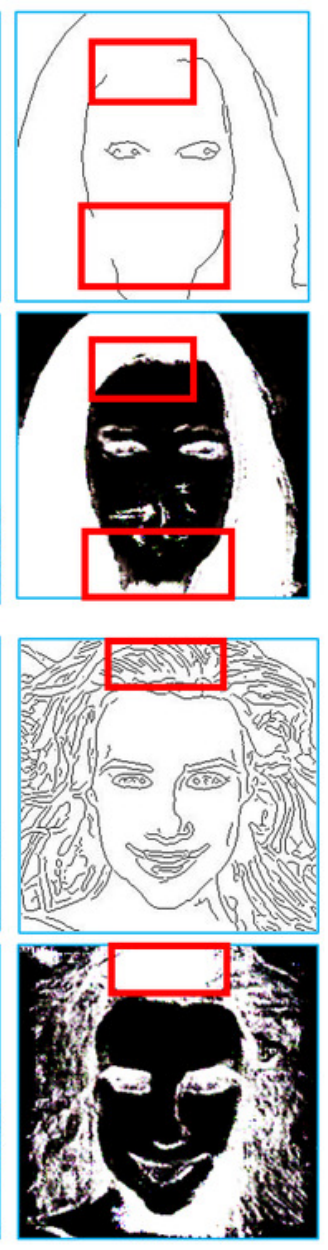
Figure 7

Comparison of different edge detectors: (a) results of Canny. (b) results of Sobel. (c) results of Laplace. (d) results of Gradient.

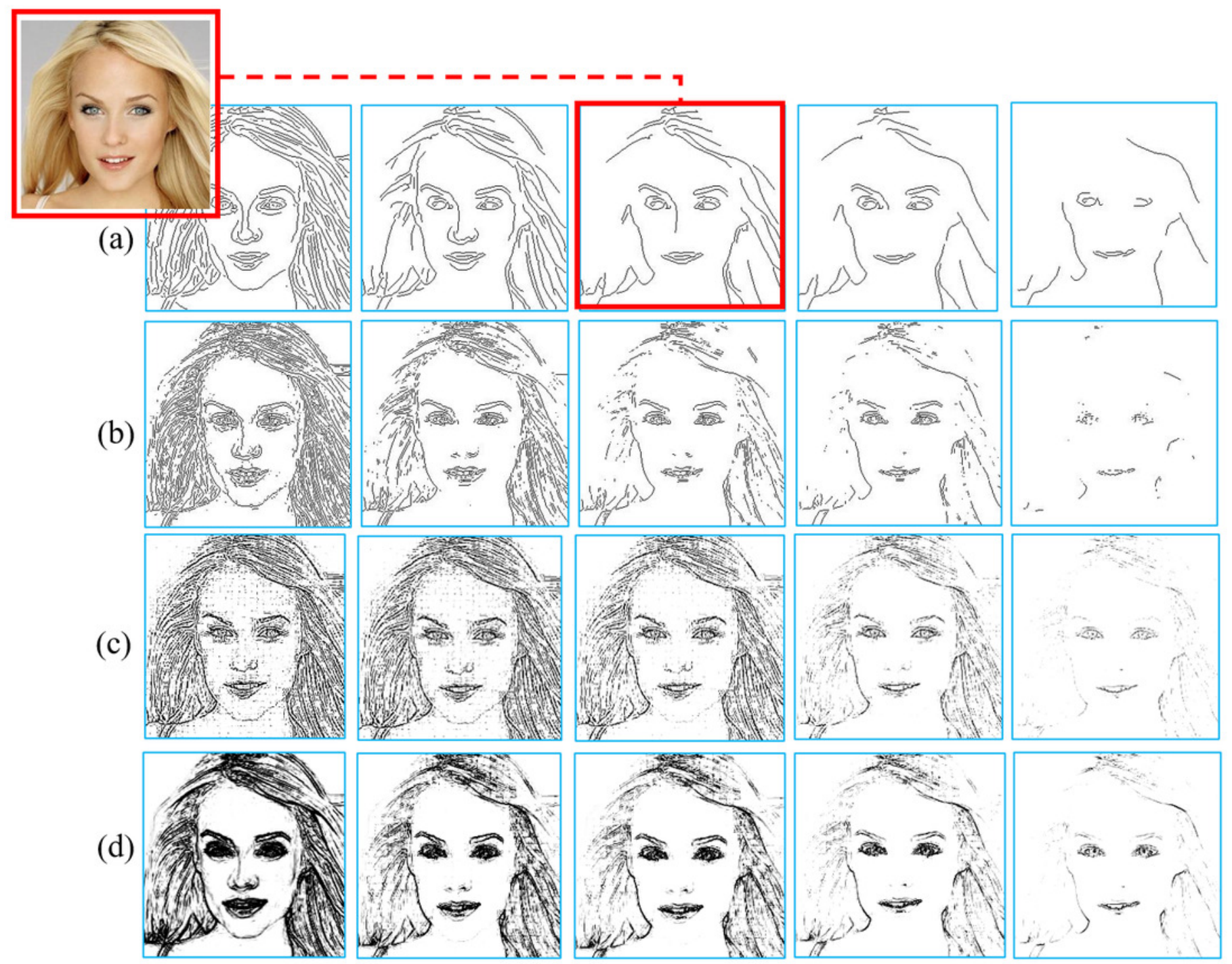




\section{Figure 8}

Inference results with examples of refined images and final outputs.

The red boxes represent blending areas in the refined region, which can be reflected by the brightness in the generated image outputs.
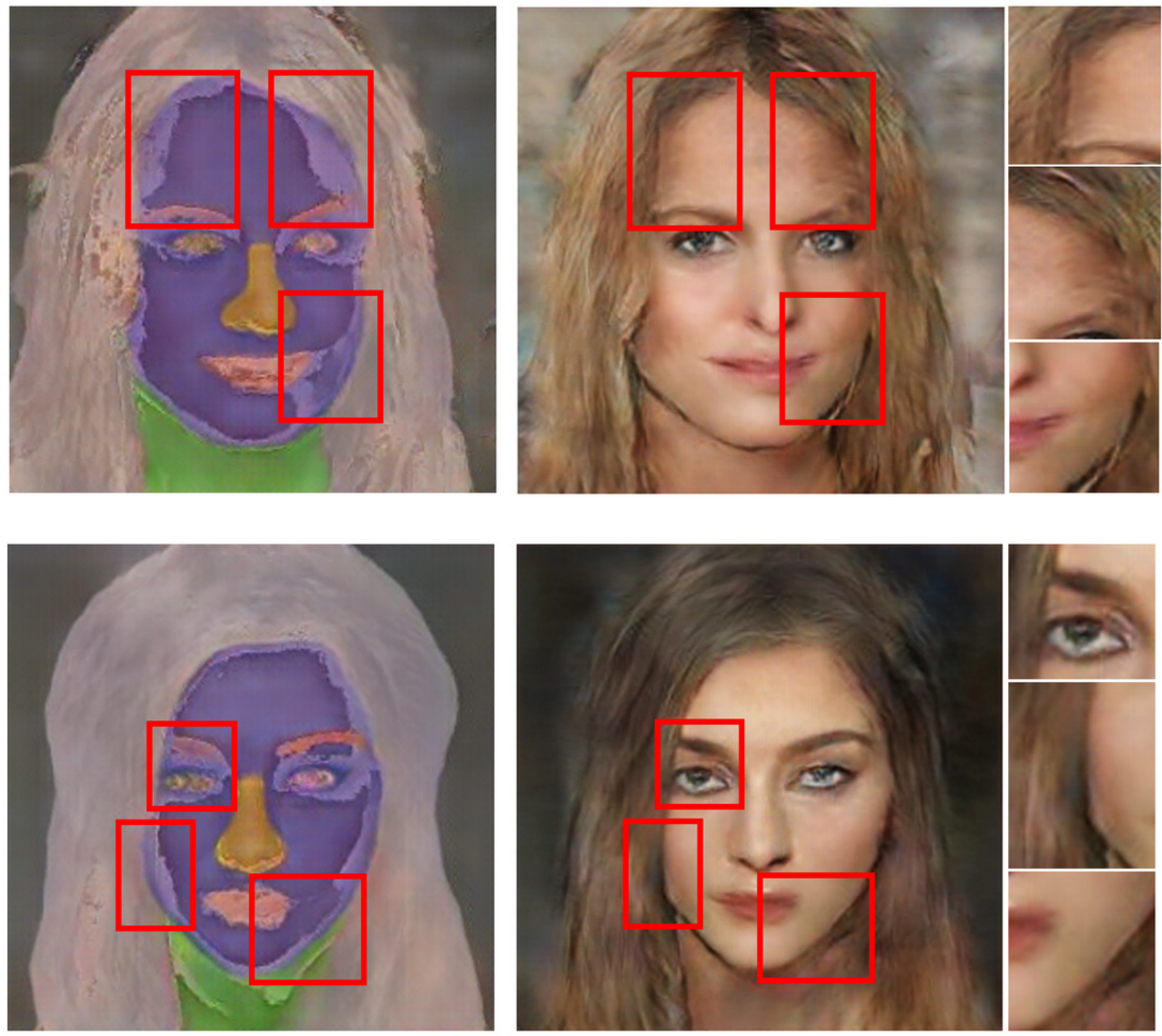
Figure 9

Results from exchanging conditional facial edges to generate diverse styles of facial images.

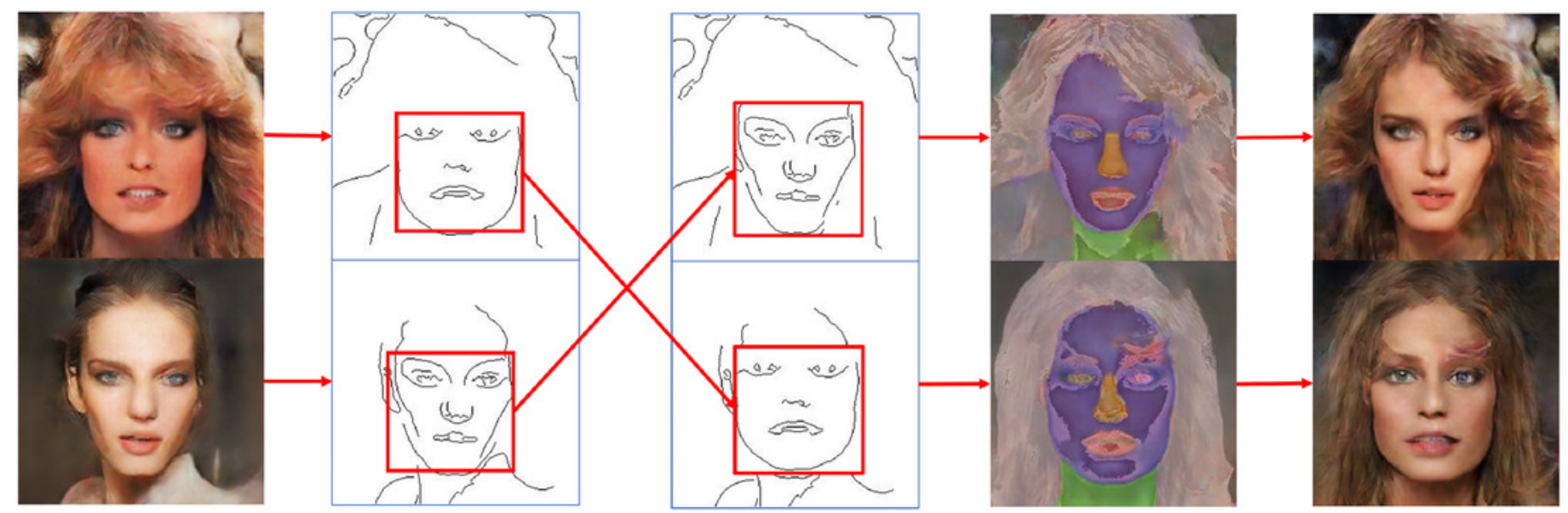




\section{Figure 10}

Inference results with example images in the source, interim, and target domains respectively.

The various density levels in the conditional inputs were not included in the training phase

except the one in the red box generated by Canny edge detector with threshold ratio $=0.4$. The results are from GANs trained using 50 images only. 


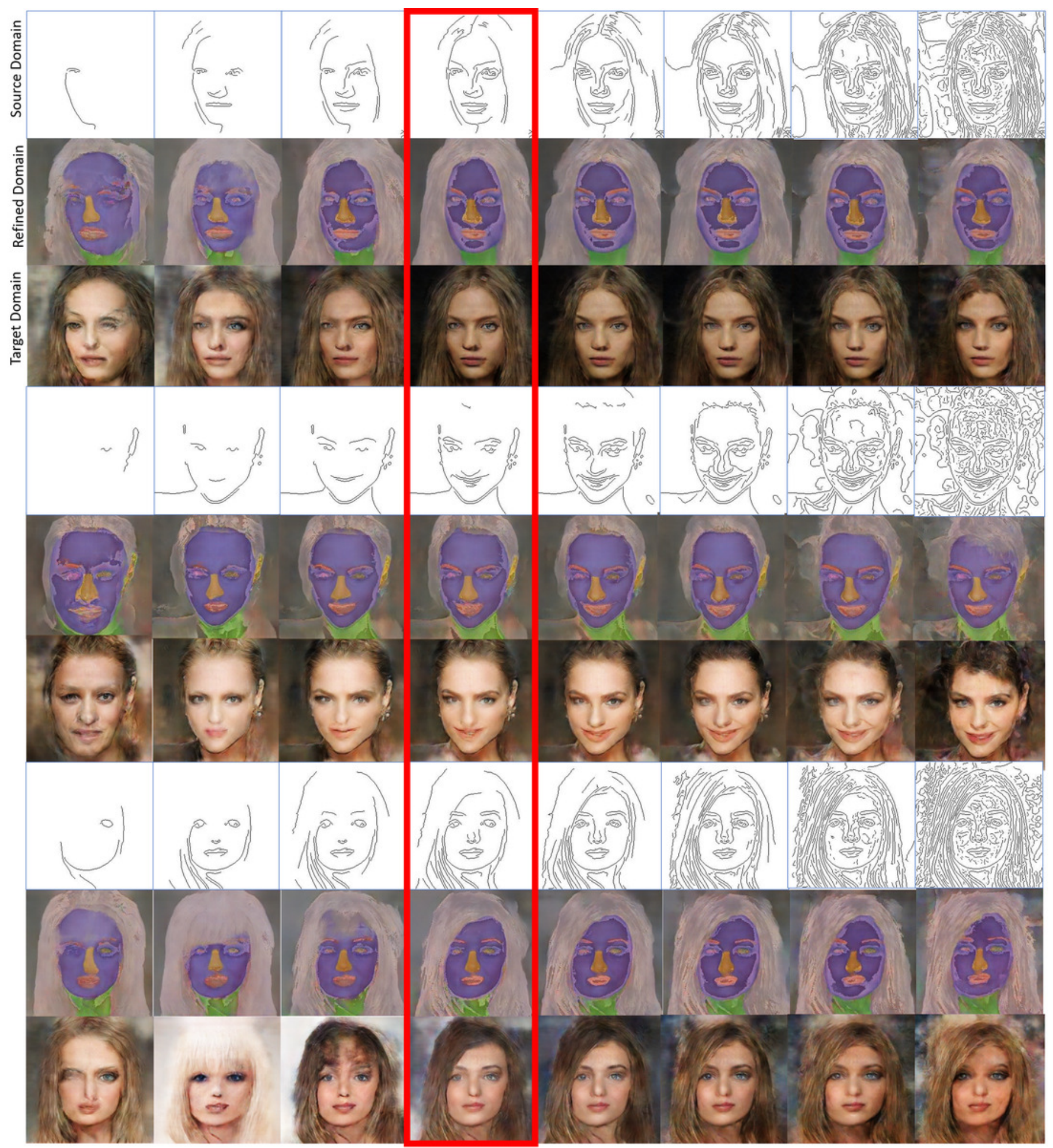




\section{Figure 11}

Examples of facial image augmentation results using 50 training images, with parts of input edges modified for introducing diversity so as to augment each training image with desirable facial features.
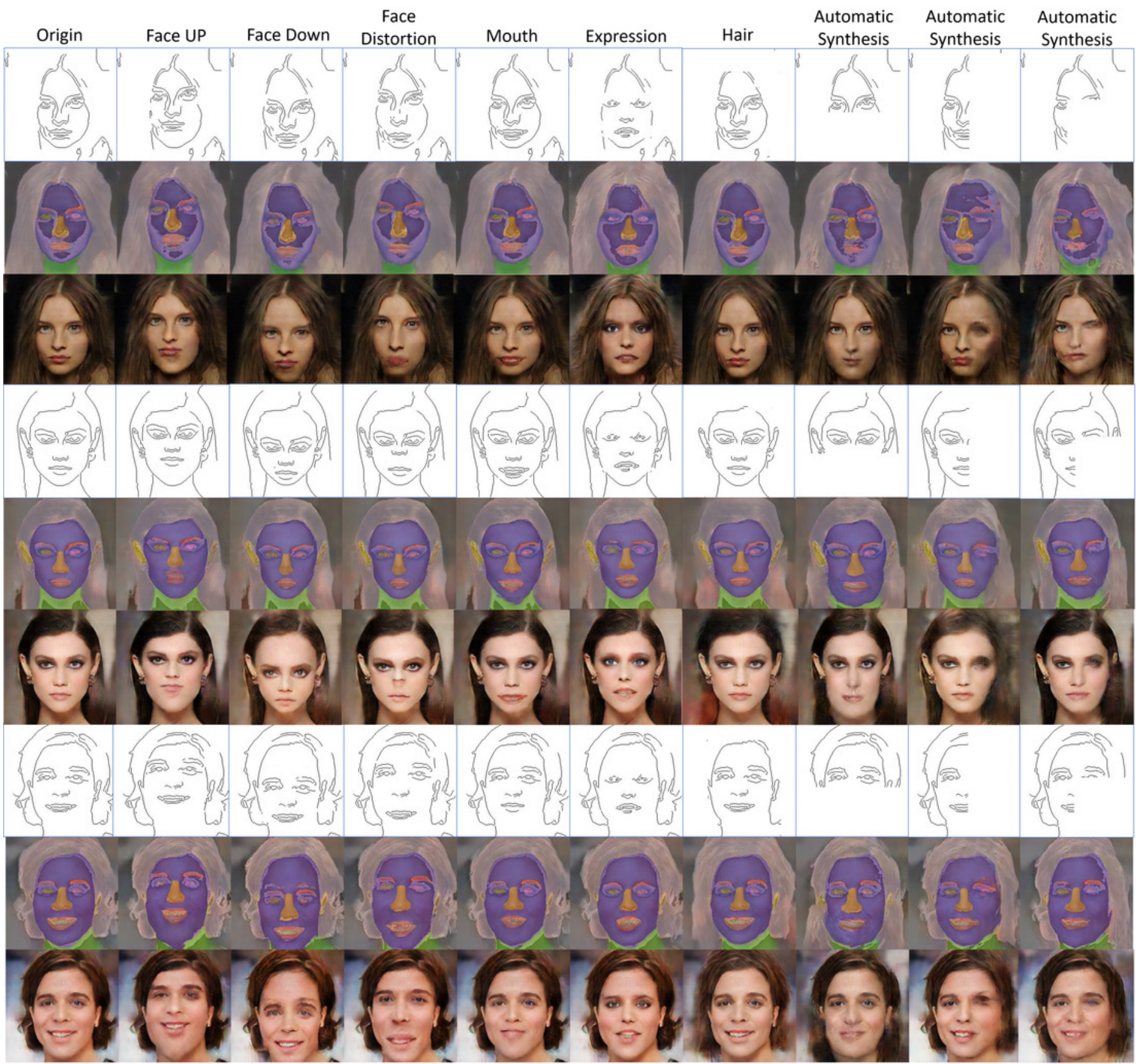


\section{Figure 12}

Examples of facial image augmentation results using 50 training images, with face components and hairstyles in different training images swapped in the edges as conditional inputs so as to generate diverse facial images. 
Facial Components

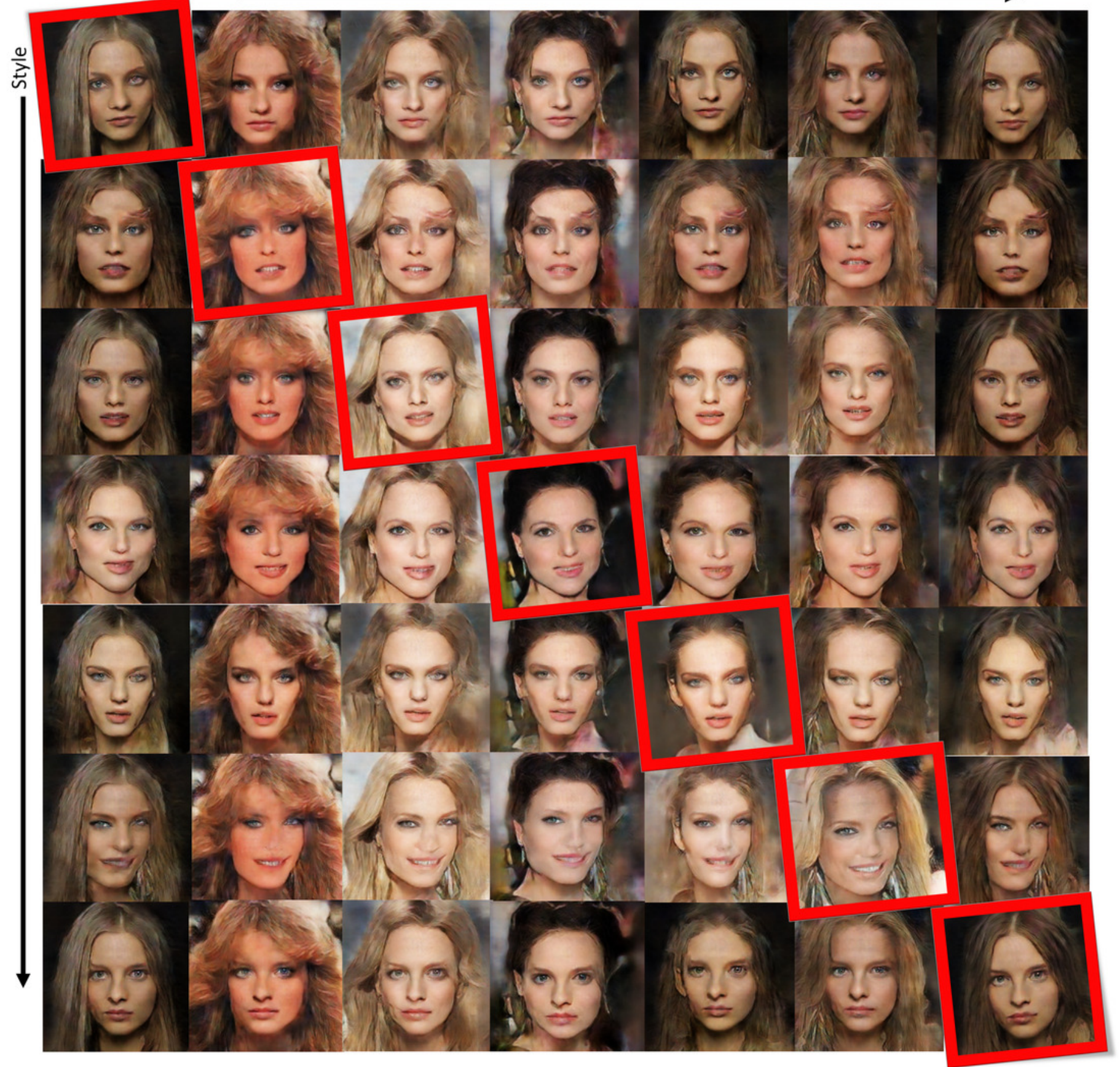




\section{Figure 13}

Inference results with example images in the source, interim, and target domains respectively.

The conditional inputs are hand-drawn sketches showing different facial expressions. The proposed conditional GAN was trained using 50 training images.
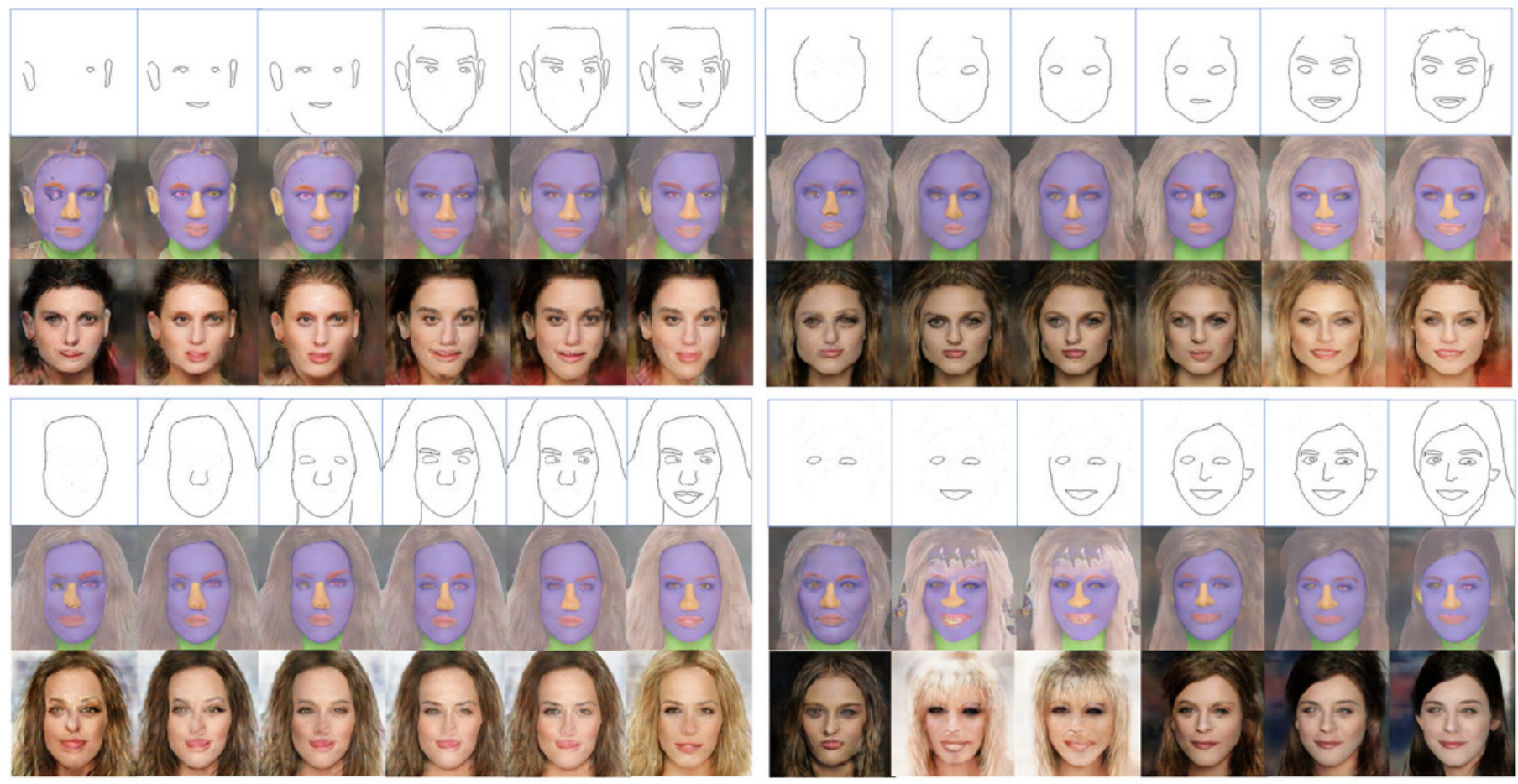


\section{Figure 14}

Inference results generated with sparse edge inputs (the first row), in comparison with those obtained from the state-of-the-art conditional GANs.

The images were generated respectively by the three GANs for comparison, trained using the same small dataset of 50 training images.
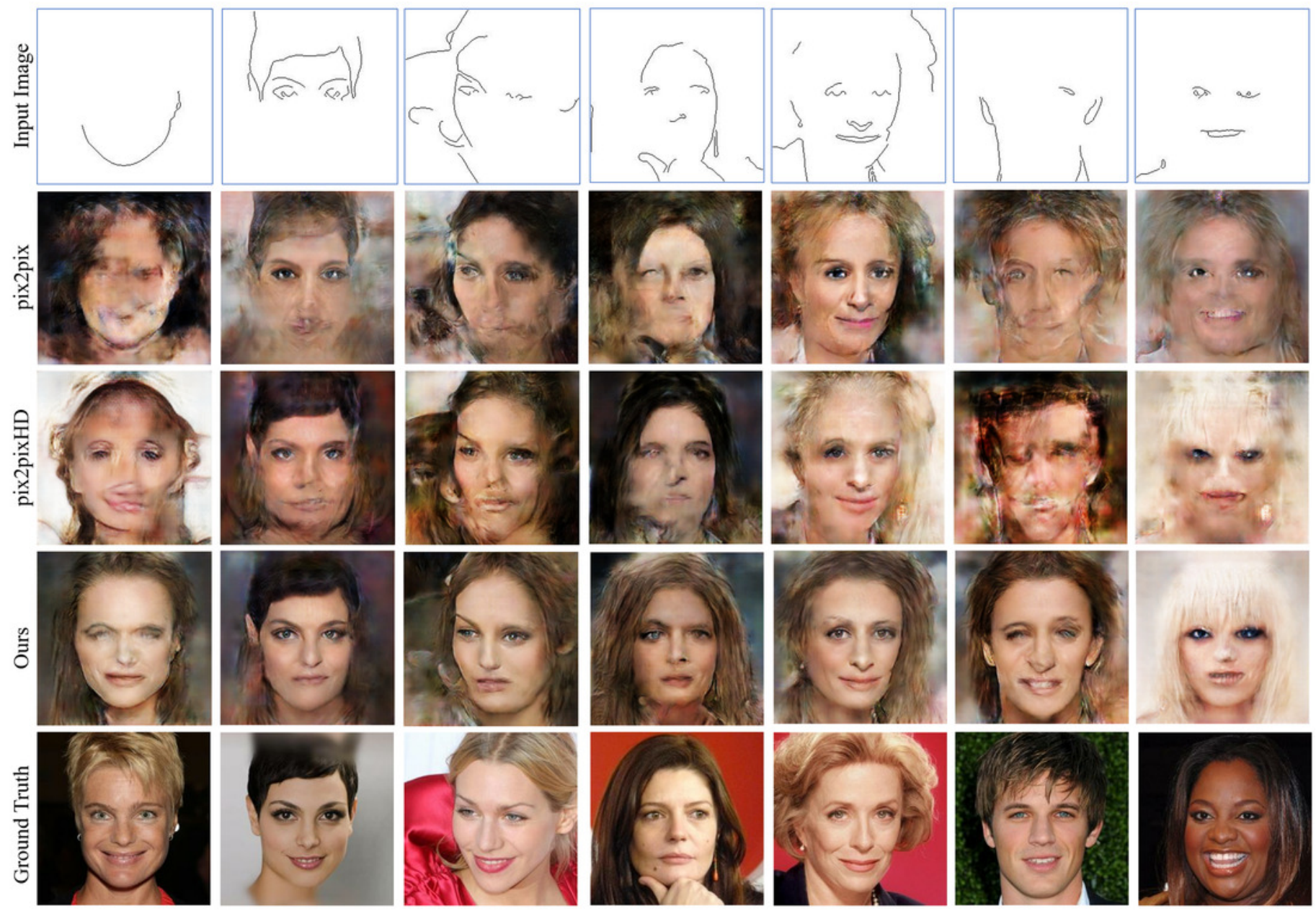


\section{Figure 15}

FID and KID scores of double U-nets with an interim domain and single U-net with different levels of input edge density respectively.

One input type (threshold ratio $=0.4$ ) and three input types (threshold ratios $=0.2,0.4,0.6$ ) in the source domain were used respectively during training with a small training dataset of 50 images. The FID and KID scores were calculated based on the same 1000 inference images at different edge density levels.
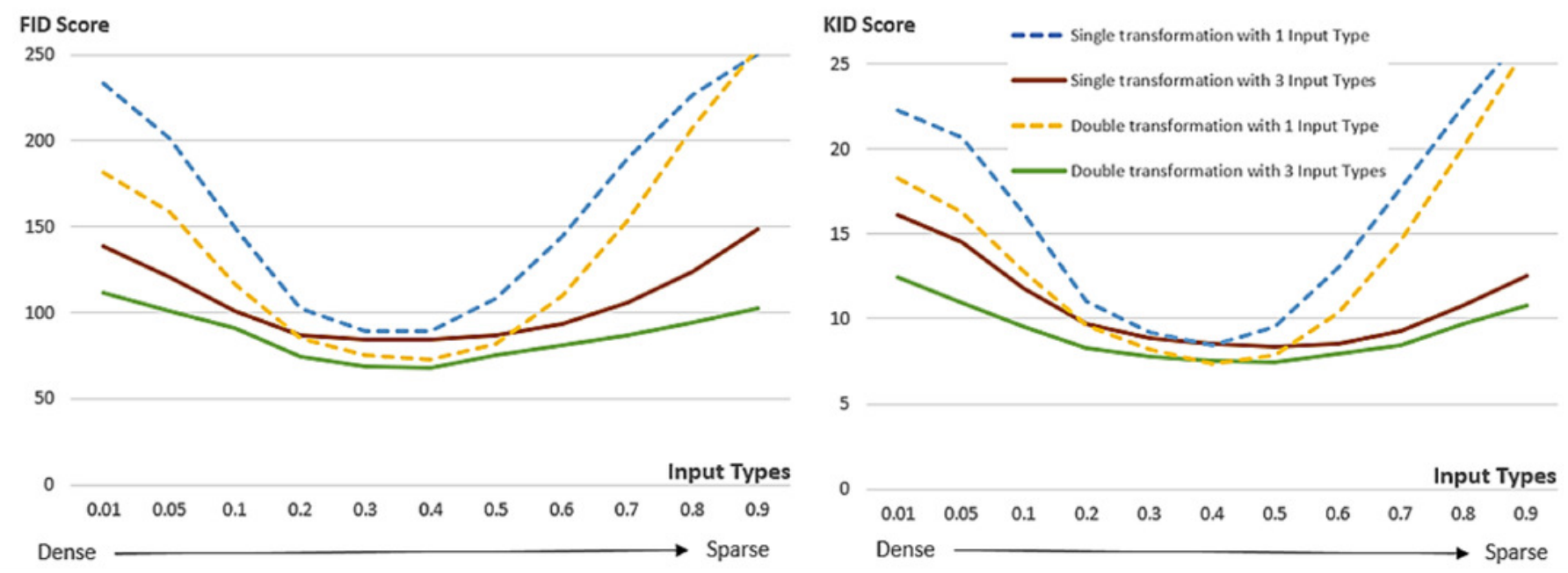
Figure 16

Changes of FID scores (first row) and KID scores (second row) with different number of training images.

Comparison among three edge-to-image translation methods with sparse and dense edge inputs respectively: pix2pix, pix2pixHD and ours.

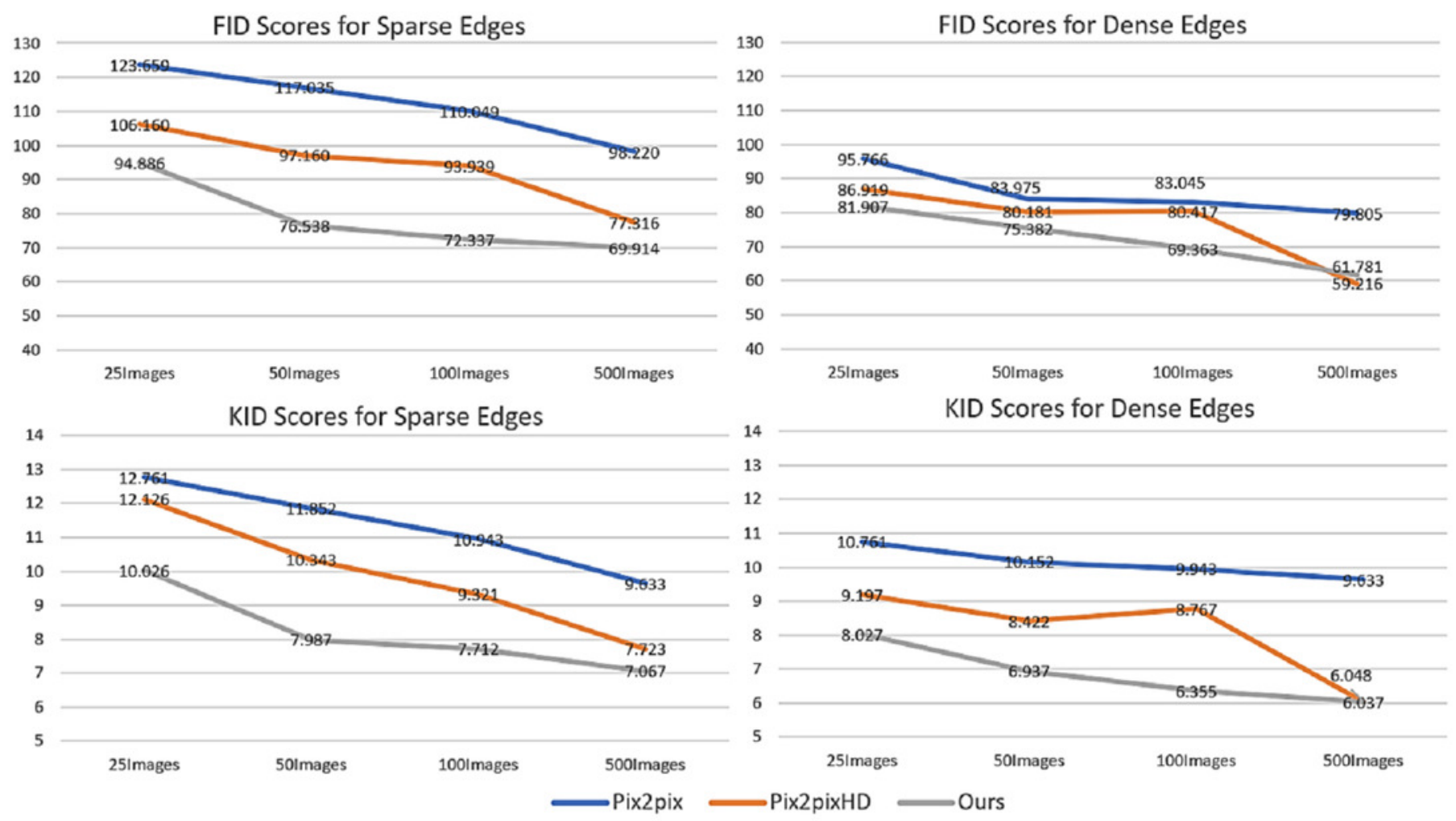

